TRANSACTIONS OF THE

AMERICAN MATHEMATICAL SOCIETY

Volume 360, Number 11, November 2008, Pages 5629-5666

S 0002-9947(08)04755-7

Article electronically published on June 19, 2008

\title{
THE DIMENSIONS OF SPACES OF HOLOMORPHIC SECOND-ORDER AUTOMORPHIC FORMS AND THEIR COHOMOLOGY
}

\author{
NIKOLAOS DIAMANTIS AND CORMAC O'SULLIVAN
}

\begin{abstract}
In this paper we answer a question of Zagier and find the dimensions of spaces of holomorphic second-order forms of even weight. We also establish a cohomological interpretation and prove an Eichler-Shimura-type isomorphism.
\end{abstract}

\section{INTRODUCTION}

In the paper [KZ], Kleban and Zagier find that the study of crossing probabilities in percolation theory leads naturally to holomorphic second-order modular forms. In this paper we answer a question posed by Zagier and compute the exact dimensions of these second-order spaces for even weight. We also establish a cohomological interpretation of these spaces which is analogous to that of Eichler and Shimura for the usual modular forms.

Second-order automorphic forms have recently arisen in several other contexts besides percolation theory, for example Eisenstein series with modular symbols in the papers $\mathrm{G}$, O1], $\mathrm{PR}$ and GL(2) converse theorems in $[\mathrm{F},[\mathrm{FW}$ and $[\mathrm{IM}$. In CDO, classification theorems for smooth second-order forms were provided, but we were only able to prove an upper bound for the dimensions of the holomorphic spaces. Here we establish a formula for the dimension confirming that it equals the upper bound given in [CDO] when the weight is not 2 . The weight 2 case was the hardest to resolve, requiring the analytic continuation of a number of related series. Interestingly, in this case the dimension differs from the natural upper bound by 1. These results may have consequences for some percolation theory problems and the above mentioned converse theorems.

We use the knowledge of the dimensions to express the second-order spaces in terms of Eichler cohomology. As in the classical theorem of Eichler and Shimura, the isomorphism is given explicitly by period polynomials, thereby maintaining the connection of such maps with values of (twisted) $L$-functions. We expect this isomorphism will yield applications analogous to those of the Eichler-Shimura isomorphism. We also hope it will help us in obtaining a natural geometric interpretation for second-order automorphic forms.

Received by the editors February 24, 2005.

2000 Mathematics Subject Classification. Primary 11F12; Secondary 11F72, 11F75.

The first author was partially supported by EPSRC grant EP/D032350/1.

The second author was partially supported by a grant from the City University of New York PSC-CUNY Research Award Program. 


\section{Definitions AND STATEMENT OF MAIN RESUlts}

Let $\Gamma \subset P S L_{2}(\mathbb{R})$ be a Fuchsian group of the first kind acting on the upper half plane $\mathfrak{H}$ with non-compact quotient $\Gamma \backslash \mathfrak{H}$. As usual we write $x+i y=z \in \mathfrak{H}$. Let $d \mu z$ be the hyperbolic volume form $d x d y / y^{2}$ and $V$ the volume of $\Gamma \backslash \mathfrak{H}$. For a fundamental domain $\mathfrak{F}$, fix representatives of the inequivalent cusps in $\overline{\mathfrak{F}}$ and give them labels such as $\mathfrak{a}, \mathfrak{b}$. Use the corresponding scaling matrices $\sigma_{\mathfrak{a}}, \sigma_{\mathfrak{b}}$ to give convenient local coordinates near these cusps as in [11, Chapter 2] for example. The subgroup $\Gamma_{\mathfrak{a}}$ is the set of elements of $\Gamma$ fixing $\mathfrak{a}$ and

$$
\sigma_{\mathfrak{a}}{ }^{-1} \Gamma_{\mathfrak{a}} \sigma_{\mathfrak{a}}=\Gamma_{\infty}=\left\{ \pm\left(\begin{array}{cc}
1 & m \\
0 & 1
\end{array}\right) \mid m \in \mathbb{Z}\right\} .
$$

The slash operator $\left.\right|_{k}$ defines an action of $P S L_{2}(\mathbb{R})$ on functions $f: \mathfrak{H} \mapsto \mathbb{C}$ by

$$
\left(\left.f\right|_{k} \gamma\right)(z)=f(\gamma z)(c z+d)^{-k}
$$

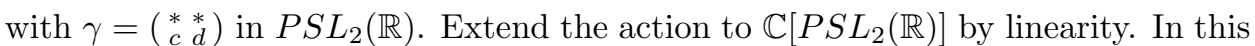
context we set $j(\gamma, z)=c z+d$ for later use.

We shall also require the generators of the group $\Gamma$. Suppose $\Gamma \backslash \mathfrak{H}$ has genus $g, r$ elliptic fixed points and $p$ cusps. Then there are $2 g$ hyperbolic elements $\gamma_{i}$, $r$ elliptic elements $\epsilon_{i}$ and $p$ parabolic elements $\pi_{i}$ generating $\Gamma$ and satisfying the $r+1$ relations:

$$
\left[\gamma_{1}, \gamma_{g+1}\right] \ldots\left[\gamma_{g}, \gamma_{2 g}\right] \epsilon_{1} \ldots \epsilon_{r} \pi_{1} \ldots \pi_{p}=1, \quad \epsilon_{j}^{e_{j}}=1
$$

for $1 \leqslant j \leqslant r$ and integers $e_{j} \geqslant 2$. Here $[a, b]$ denotes the commutator $a b a^{-1} b^{-1}$ of $a, b$ (cf. [I1], Proposition 2.6).

Let $k$ be an integer. As explained below, a first or second-order form $f$ is defined from the following list of alternatives. It may satisfy either

H. $f: \mathfrak{H} \rightarrow \mathbb{C}$ is holomorphic or

S. $f: \mathfrak{H} \rightarrow \mathbb{C}$ is smooth.

Its automorphy condition may involve, for some vector space of functions $V$,

$\left.\mathbf{A}(\mathrm{V}) \cdot f\right|_{k}(\gamma-1) \in V$ for all $\gamma$ in $\Gamma$ or

P. $\left.f\right|_{k}(\pi-1)=0$ for all parabolic $\pi$ in $\Gamma$.

Finally, we need to impose growth conditions which may be cuspidal $(\mathbf{C})$, or noncuspidal $(\mathbf{N})$ :

C. for each cusp $\mathfrak{a},\left(\left.f\right|_{k} \sigma_{\mathfrak{a}}\right)(z) \ll e^{-c y}$ as $y \rightarrow \infty$ uniformly in $x$ for some constant $c>0$ or

N. for each cusp $\mathfrak{a},\left(\left.f\right|_{k} \sigma_{\mathfrak{a}}\right)(z) \ll y^{c}$ as $y \rightarrow \infty$ uniformly in $x$ for some constant $c$.

Define $S_{k}(\Gamma)$, the space of holomorphic, weight $k$, cusp forms for $\Gamma$, to be the $\mathbb{C}$-vector space of functions $f$ such that $\mathbf{H}, \mathbf{A}(0)$ and $\mathbf{C}$ hold. The corresponding space of modular forms, $M_{k}(\Gamma)$, satisfies $\mathbf{H}, \mathbf{A}(0)$ and $\mathbf{N}$. Both these spaces are always finite dimensional. Using the Riemann-Roch theorem, exact formulas for their dimensions are given in $[\mathbf{S h}$, Theorems 2.23, 2.24 (see Section 4). Following DKMO, the space $S_{k}^{2}(\Gamma)$ of holomorphic, weight $k$, second-order cusp forms consists of functions satisfying $\mathbf{H}, \mathbf{A}\left(S_{k}(\Gamma)\right), \mathbf{P}$ and $\mathbf{C}$. This new space is similar to $S_{k}(\Gamma)$, the only difference being the weaker automorphy rule. Naturally, we define the space $M_{k}^{2}(\Gamma)$ with the conditions $\mathbf{H}, \mathbf{A}\left(M_{k}(\Gamma)\right), \mathbf{P}$ and $\mathbf{N}$. Lastly, replacing $\mathbf{H}$ with $\mathbf{S}$, we obtain the smooth spaces $\tilde{S}_{k}(\Gamma), \tilde{M}_{k}(\Gamma), \tilde{S}_{k}^{2}(\Gamma)$ and $\tilde{M}_{k}^{2}(\Gamma)$ (with $\mathbf{A}\left(\tilde{S}_{k}(\Gamma)\right)$ and $\mathbf{A}\left(\tilde{M}_{k}(\Gamma)\right)$ in the definitions of these last two). They are infinite 
dimensional in general. Restricting to certain eigenspaces of the Laplacian does yield finite dimensional spaces; see [CDO].

To summarize, the main holomorphic spaces used subsequently are:
- $S_{k}(\Gamma)$ defined with
- $S_{k}^{2}(\Gamma)$ defined with
- $R_{k}^{2}(\Gamma)$ defined with
- $M_{k}(\Gamma)$ defined with
- $M_{k}^{2}(\Gamma)$ defined with
$\mathbf{H}, \mathbf{A}(0) \quad$ and $\mathbf{C}$,
$\mathbf{H}, \mathbf{A}\left(S_{k}(\Gamma)\right), \mathbf{P}$ and $\mathbf{C}$,
$\mathbf{H}, \mathbf{A}\left(S_{k}(\Gamma)\right), \mathbf{P}$ and $\mathbf{N}$,
$\mathbf{H}, \mathbf{A}(0) \quad$ and $\mathbf{N}$,
$\mathbf{H}, \quad \mathbf{A}\left(M_{k}(\Gamma)\right), \mathbf{P}$ and $\mathbf{N}$.

We included the space $R_{k}^{2}(\Gamma)$ above, which has some interesting elements as we shall see in Section 5. Their smooth counterparts are:

- $\tilde{S}_{k}(\Gamma)$ defined with $\mathbf{S}, \quad \mathbf{A}(0) \quad$ and $\mathbf{C}$,

- $\tilde{S}_{k}^{2}(\Gamma)$ defined with $\mathbf{S}, \quad \mathbf{A}\left(\tilde{S}_{k}(\Gamma)\right), \mathbf{P}$ and $\mathbf{C}$,

- $\tilde{M}_{k}(\Gamma)$ defined with $\mathbf{S}, \quad \mathbf{A}(0) \quad$ and $\mathbf{N}$,

- $\tilde{M}_{k}^{2}(\Gamma)$ defined with $\mathbf{S}, \quad \mathbf{A}\left(\tilde{M}_{k}(\Gamma)\right), \mathbf{P}$ and $\mathbf{N}$.

In the paper DKMO we examine the effects of weakening or altering the conditions defining each of these spaces. Here we prove three main results.

Theorem 2.1. For $k$ in $2 \mathbb{Z}$ and $\Gamma \backslash \mathfrak{H}$ non-compact with genus $g$, we have

$$
\begin{aligned}
& \operatorname{dim} S_{k}^{2}(\Gamma)=0 \text { if } k \leqslant 0, \\
& \operatorname{dim} S_{2}^{2}(\Gamma)=\left\{\begin{array}{l}
0 \text { if } \operatorname{dim} S_{2}(\Gamma)=0, \\
(2 g+1) \operatorname{dim} S_{2}(\Gamma)-1 \text { otherwise, }
\end{array}\right. \\
& \operatorname{dim} S_{k}^{2}(\Gamma)=(2 g+1) \operatorname{dim} S_{k}(\Gamma) \text { if } k \geqslant 4 .
\end{aligned}
$$

Theorem 2.2. For $k$ in $2 \mathbb{Z}$ and $\Gamma \backslash \mathfrak{H}$ non-compact with genus $g$, we have

$$
\begin{aligned}
& \operatorname{dim} M_{k}^{2}(\Gamma)=0 \text { if } k \leqslant-2, \\
& \operatorname{dim} M_{0}^{2}(\Gamma)=g+1, \\
& \operatorname{dim} M_{k}^{2}(\Gamma)=(2 g+1) \operatorname{dim} M_{k}(\Gamma) \text { if } k \geqslant 2 .
\end{aligned}
$$

Theorem 2.3. We have the isomorphisms

$$
\begin{aligned}
& \frac{M_{2}^{2}(\Gamma)}{M_{2}(\Gamma)} \oplus \frac{\bar{S}_{2}^{2}(\Gamma)}{\bar{S}_{2}(\Gamma)} \oplus \mathbb{C} \cong H_{!}^{1}(\Gamma, \mathbb{C}), \\
& \frac{M_{k}^{2}(\Gamma)}{M_{k}(\Gamma)} \oplus \frac{\bar{S}_{k}^{2}(\Gamma)}{\bar{S}_{k}(\Gamma)} \cong H_{!}^{1}\left(\Gamma, P_{k-2}\right) \text { for even } k \geqslant 4,
\end{aligned}
$$

where $P_{k-2}$ is the space of all polynomials of degree at most $k-2$ and $H_{!}^{1}(\Gamma, \mathbb{C})$, $H_{!}^{1}\left(\Gamma, P_{k-2}\right)$ are associated cohomology groups as defined in Section $\%$.

We note that Goldfeld considered certain subspaces of $S_{k}^{2}(\Gamma)$ and $M_{k}^{2}(\Gamma)$ in G] (preceding the work in [KZ]) and calculated their dimensions. See also DO, Proposition 15] for a further generalization.

The proofs of Theorems 2.1 and 2.2 are constructive in that, in each case, a basis of second-order Poincaré series is given. In the weight 2 case this relies on the analytic continuation of various series. An example of the type of results we prove 
is the following. For $m \in \mathbb{Z}$ and non-negative, define the non-holomorphic, weight $k$ Poincaré series

$$
U_{\mathfrak{a} m}(z, s, k)=\sum_{\gamma \in \Gamma_{\mathfrak{a}} \backslash \Gamma} \operatorname{Im}\left({\sigma_{\mathfrak{a}}}^{-1} \gamma z\right)^{s} e\left(m{\sigma_{\mathfrak{a}}}^{-1} \gamma z\right) \varepsilon\left({\sigma_{\mathfrak{a}}}^{-1} \gamma, z\right)^{-k}
$$

for $\operatorname{Re}(s)>1$, where $\varepsilon(\gamma, z)$ is the 'non-holomorphic' weight factor $j(\gamma, z) /|j(\gamma, z)|$ and, as usual, $e(z)=e^{2 \pi i z}$.

Proposition 2.4. For $k \in 2 \mathbb{Z}, m>0$ and some $\delta>0$, the Poincaré series $U_{\mathfrak{a} m}(z, s, k)$ has a continuation to an analytic function for all $s$ with $\operatorname{Re}(s)>1-\delta$. We have

$$
U_{\mathfrak{a} m}(z, s, k) \ll y_{\Gamma}(z)^{1 / 2}
$$

for these $s$ with the implied constant depending on $s, m, k$ and $\Gamma$.

Here $y_{\Gamma}(z)$ is the invariant height

$$
y_{\Gamma}(z)=\max _{\mathfrak{a}}\left(\max _{\gamma \in \Gamma}\left(\operatorname{Im}\left(\sigma_{\mathfrak{a}}{ }^{-1} \gamma z\right)\right)\right) .
$$

3. UPPER BOUNDS FOR $\operatorname{dim} S_{k}^{2}(\Gamma)$ AND $\operatorname{dim} M_{k}^{2}(\Gamma)$

Let $\operatorname{Hom}_{0}(\Gamma, \mathbb{C})$ be the space of homomorphisms from $\Gamma$ to $\mathbb{C}$ that are zero on parabolic elements of $\Gamma$. According to a special case of the Eichler-Shimura isomorphism (see Section 7 for a complete statement), for any such homomorphism $L$ there exist unique $l_{1}, l_{2}$ in $S_{2}(\Gamma)$ so that

$$
L(\gamma)=\int_{z}^{\gamma z} l_{1}(w) d w+\overline{\int_{z}^{\gamma z} l_{2}(w) d w}
$$

for all $\gamma$ in $\Gamma$. We define the modular symbol

$$
\langle\gamma, l\rangle=\int_{z}^{\gamma z} l(w) d w
$$

for $\gamma$ in $\Gamma$ and $l$ in $S_{2}(\Gamma)$. The value of $\langle\gamma, l\rangle$ is independent of the choice of base point $z$ in $\mathfrak{H}$. For $1 \leqslant i \leqslant 2 g$ we may define

$$
\Lambda_{i}(z)=\int_{z_{0}}^{z} \lambda_{i}(w) d w+\overline{\int_{z_{0}}^{z} \mu_{i}(w) d w}
$$

for certain $\lambda_{i}, \mu_{i}$ in $S_{2}(\Gamma)$ and $z_{0}$ an arbitrary fixed element of $\mathfrak{H}$ (usually taken to be the imaginary number $i$ or $i \infty$ ) to satisfy

$$
\Lambda_{i}\left(\gamma_{j} z\right)-\Lambda_{i}(z)=\delta_{i j}
$$

for the hyperbolic generators and also

$$
\Lambda_{i}(\gamma z)-\Lambda_{i}(z)=0
$$

for the other parabolic and elliptic generators $\gamma$ of $\Gamma$. Thus $\left\{L_{i}\right\}_{i=1}^{2 g}$ defined by

$$
L_{i}(\gamma)=\Lambda_{i}(\gamma z)-\Lambda_{i}(z)
$$

forms a natural basis for $\operatorname{Hom}_{0}(\Gamma, \mathbb{C})$ dual to our choice of generators for $\Gamma$.

From the map

$$
f \mapsto\left(\left.f\right|_{k}\left(\gamma_{1}-1\right), \ldots,\left.f\right|_{k}\left(\gamma_{2 g}-1\right)\right)=\left(f_{1}, \ldots, f_{2 g}\right),
$$


it may be shown, as in DKMO, Theorem 4] that any element $f$ of $S_{k}^{2}(\Gamma)$ must satisfy

$$
f(z)=\sum_{i=1}^{2 g} f_{i}(z) \Lambda_{i}(z)+\phi(z),
$$

where each $f_{i}=\left.f\right|_{k}\left(\gamma_{i}-1\right)$ is in $S_{k}(\Gamma)$ and $\phi$ is in $\tilde{S}_{k}(\Gamma)$. Apply $\frac{d}{d \bar{z}}$ to both sides of (3.5) to get

$$
\frac{d}{d \bar{z}} \phi=-\sum_{i=1}^{2 g} f_{i} \overline{\mu_{i}} .
$$

So $\phi$, if it exists, is uniquely specified by (3.6) up to addition of an element of $S_{k}(\Gamma)$. Counting the degrees of freedom in (3.5) shows that, for $k \in \mathbb{Z}$,

$$
\operatorname{dim} S_{k}^{2}(\Gamma) \leqslant(2 g+1) \operatorname{dim} S_{k}(\Gamma) .
$$

Similarly, $f$ in $M_{k}^{2}(\Gamma)$ satisfies (3.5) with $f_{i}$ in $M_{k}(\Gamma)$ and $\phi$ in $\tilde{M}_{k}(\Gamma)$. Thus

$$
\operatorname{dim} M_{k}^{2}(\Gamma) \leqslant(2 g+1) \operatorname{dim} M_{k}(\Gamma) .
$$

In fact the dimensions of these spaces, for weight $k \neq 2$, are given by the upper bounds in (3.7) and (3.8). We will show this in the next section using an extension of the usual Poincaré series construction to build linearly independent second-order forms. The case of weight $k=2$ must be singled out for special attention since the Poincaré series we need are no longer absolutely convergent. An analysis in Sections 5 and 6 shows that our constructions come up one short of the upper bounds at this weight.

Before discussing Poincaré series, we note that there is a much easier way to find elements of $S_{k}^{2}(\Gamma)$ and $M_{k}^{2}(\Gamma)$. Certainly we have the subspaces $S_{k}(\Gamma) \subset S_{k}^{2}(\Gamma)$ and $M_{k}(\Gamma) \subset M_{k}^{2}(\Gamma)$. Also, it may be checked (see Lemma 4.1 in the next section) that any product

$$
f(z) \int_{z_{0}}^{z} h(w) d w
$$

for $f$ in $S_{k}(\Gamma)$ and $h$ in $S_{2}(\Gamma)$ gives an element of $S_{k}^{2}(\Gamma)$. If $f$ is in $M_{k}(\Gamma)$, then (3.9) yields an element of $M_{k}^{2}(\Gamma)$. This suggests the natural decomposition:

$$
\begin{aligned}
S_{k}^{2}(\Gamma) & =S_{k}^{2}(\Gamma)^{+} \oplus S_{k}(\Gamma) \oplus S_{k}^{2}(\Gamma)^{-}, \\
M_{k}^{2}(\Gamma) & =M_{k}^{2}(\Gamma)^{+} \oplus M_{k}(\Gamma) \oplus M_{k}^{2}(\Gamma)^{-},
\end{aligned}
$$

where the spaces $S_{k}^{2}(\Gamma)^{+}, M_{k}^{2}(\Gamma)^{+}$consist of all linear combinations of elements of the form (3.9). In this way, for $k \geqslant 0$, we get the simple lower bounds

$$
\begin{gathered}
(g+1) \operatorname{dim} S_{k}(\Gamma)=\operatorname{dim}\left(S_{k}^{2}(\Gamma)^{+} \oplus S_{k}(\Gamma)\right) \leqslant \operatorname{dim} S_{k}^{2}(\Gamma), \\
(g+1) \operatorname{dim} M_{k}(\Gamma)=\operatorname{dim}\left(M_{k}^{2}(\Gamma)^{+} \oplus M_{k}(\Gamma)\right) \leqslant \operatorname{dim} M_{k}^{2}(\Gamma) .
\end{gathered}
$$

A second-order form similar to (3.9) appears in the work of Kleban and Zagier, [KZ], on percolation theory. For the Dedekind eta function $\eta(z)$ we have

$$
\begin{gathered}
\eta(z)^{4} \in S_{2}\left(\Gamma_{\theta}, \chi\right), \\
\eta(z / 2)^{8} \eta(2 z)^{8} \eta(z)^{-12} \in S_{2}\left(\Gamma_{\theta}, \bar{\chi}\right)
\end{gathered}
$$


for $\Gamma_{\theta}$ the subgroup of $P S L_{2}(\mathbb{Z})$ generated by $\pm\left(\begin{array}{cc}1 & 2 \\ 0 & 1\end{array}\right), \pm\left(\begin{array}{cc}0 & -1 \\ 1 & 0\end{array}\right)$. Here $\chi$ is a certain character for $\Gamma_{\theta}$. They show in equations (16), (19) of [KZ that the derivative of a certain crossing probability for a rectangle with aspect ratio $r$ is

$$
K(z)=\frac{-16 \pi i}{\sqrt{3}} \eta(z)^{4} \int_{i \infty}^{z} \eta(w / 2)^{8} \eta(2 w)^{8} \eta(w)^{-12} d w
$$

at $z=i r$. Consult $[\mathrm{KZ}$ for details. The expression $K(z)$ is a type of second-order form satisfying $\left.K\right|_{2}(\gamma-1) \in S_{2}\left(\Gamma_{\theta}, \chi\right)$ for all $\gamma$ in $\Gamma_{\theta}$, and conditions $\mathbf{H}$ and $\mathbf{C}$.

\section{Exact Dimensions of $S_{k}^{2}(\Gamma)$ AND $M_{k}^{2}(\Gamma)$ FOR $k>2$}

First we look at the spaces $S_{k}(\Gamma)$ and $M_{k}(\Gamma)$ with $k \in 2 \mathbb{Z}$. Recall that $g$ is the genus of $\Gamma \backslash \mathfrak{H}$ and $p$ the number of inequivalent cusps. By Theorems 2.23 and 2.24 of [Sh] we have the following:

$$
\begin{aligned}
\operatorname{dim} S_{k}(\Gamma) & =\operatorname{dim} M_{k}(\Gamma)=0 \text { for } k<0, \\
\operatorname{dim} S_{0}(\Gamma) & =\left\{\begin{array}{l}
1 \text { if } p=0, \\
0 \text { if } p>0,
\end{array}\right. \\
\operatorname{dim} S_{2}(\Gamma) & =g, \\
\operatorname{dim} M_{0}(\Gamma) & =1, \\
\operatorname{dim} M_{2}(\Gamma) & =\left\{\begin{array}{l}
g \text { if } p=0, \\
g+p-1 \text { if } p>0,
\end{array}\right. \\
\operatorname{dim} M_{k}(\Gamma) & =\operatorname{dim} S_{k}(\Gamma)+p \text { if } k \geqslant 4
\end{aligned}
$$

Also for $k \geqslant 4$, with (2.1),

$$
\operatorname{dim} S_{k}(\Gamma)=(k-1)(g-1)+(k / 2-1) p+\sum_{j=1}^{r} \frac{k\left(e_{j}-1\right)}{2 e_{j}} .
$$

The analogous results for $k$ odd, $\neq 1$ also appear in [Sh, Theorem 2.25].

What are the elements of these spaces? For $k=0$ the elements of $M_{0}(\Gamma)$ (and $S_{0}(\Gamma)$ if there are no cusps) are just the constant functions. When $k \geqslant 4$ is even and $p>0, S_{k}(\Gamma)$ is spanned by the Poincaré series

$$
P_{\mathfrak{a} m}(z)=P_{\mathfrak{a} m}(z)_{k}=\sum_{\gamma \in \Gamma_{\mathfrak{a}} \backslash \Gamma} j\left(\sigma_{\mathfrak{a}}{ }^{-1} \gamma, z\right)^{-k} e\left(m \sigma_{\mathfrak{a}}{ }^{-1} \gamma z\right)
$$

with $m>0$. For example we may fix a single cusp $\mathfrak{a}$ and find a linearly independent basis with $\operatorname{dim} S_{k}(\Gamma)$ different values of $m>0$; see [2, Corollary 3.5]. The extra $p$ dimensions of $M_{k}(\Gamma)$ come from the linearly independent $P_{\mathfrak{a} 0}(z)_{k}$ as $\mathfrak{a}$ varies over the $p$ inequivalent cusps as in [Sa, Section 1.4]. When $m=0$ these series (4.1) are called the holomorphic Eisenstein series. If we let $E_{k}(\Gamma)$ denote the space of these Eisenstein series, then we have the direct sum

$$
M_{k}(\Gamma)=E_{k}(\Gamma) \oplus S_{k}(\Gamma) .
$$

Thus, varying $\mathfrak{a}$ and $m \geqslant 0$ in (4.1) and taking linear combinations produces all elements of $M_{k}(\Gamma)$ for $k \geqslant 4$. We describe what happens at weight $k=2$ later in Section 5 .

We will now prove that the dimensions of $S_{k}^{2}(\Gamma)$ and $M_{k}^{2}(\Gamma)$ attain the upper bounds (3.7) and (3.8) by producing enough second-order forms. In some cases this 
can be achieved using results of $[\mathrm{Gu}$ and $[\mathrm{K}$. However, these techniques would not yield explicit bases. To construct explicit bases we use an extension of the above Poincaré series. This idea, in different guise, appears in G]. Set

$$
P_{\mathfrak{a} m}(z, L)=P_{\mathfrak{a} m}(z, L)_{k}=\sum_{\gamma \in \Gamma_{\mathfrak{a}} \backslash \Gamma} L(\gamma) j\left(\sigma_{\mathfrak{a}}{ }^{-1} \gamma, z\right)^{-k} e\left(m \sigma_{\mathfrak{a}}{ }^{-1} \gamma z\right),
$$

for $m \geqslant 0$ and $L$ in $\operatorname{Hom}_{0}(\Gamma, \mathbb{C})$. To show that this is absolutely convergent and holomorphic for $k \geqslant 4$ we need the next lemma.

Lemma 4.1. For any $f$ in $S_{2}(\Gamma)$, all $z \in \mathfrak{H}$ and any cusp $\mathfrak{a}$,

$$
\int_{z_{0}}^{z} f(w) d w \ll\left|\log \left(\operatorname{Im}\left(\sigma_{\mathfrak{a}}{ }^{-1} z\right)\right)\right|+1
$$

with an implied constant independent of $z$.

The proof uses that $y|f(z)| \ll 1$ for any weight 2 cusp form and also that $f(z+1)=f(z)$. See [DKMO, Lemma 3] for details.

Proposition 4.2. For $L$ in $\operatorname{Hom}_{0}(\Gamma, \mathbb{C})$ and $4 \leqslant k \in 2 \mathbb{Z}$ it is the case that

$$
\begin{aligned}
& P_{\mathfrak{a} m}(z, L)_{k} \in M_{k}^{2}(\Gamma) \quad \text { if } m=0, \\
& P_{\mathfrak{a} m}(z, L)_{k} \in S_{k}^{2}(\Gamma) \quad \text { if } m>0 .
\end{aligned}
$$

Proof. For $L$ as above, all $z \in \mathfrak{H}$ and any cusp $\mathfrak{a}$, it follows from (3.1) and Lemma 4.1 that

$$
L(\gamma) \ll\left|\log \left(\operatorname{Im}\left(\sigma_{\mathfrak{a}}{ }^{-1} \gamma z\right)\right)\right|+\left|\log \left(\operatorname{Im}\left(\sigma_{\mathfrak{a}}{ }^{-1} z\right)\right)\right|+1
$$

with an implied constant independent of $z$ and $\gamma \in \Gamma$. It is also true that $|\log y|<$ $y+1 / y$ and hence $|\log y|<\varepsilon^{-1}\left(y^{\varepsilon}+y^{-\varepsilon}\right)$ for any $y>0$ and $\varepsilon>0$. Therefore

$$
\begin{aligned}
P_{\mathfrak{a} m}(z, L)_{k} \ll & \sum_{\gamma \in \Gamma_{\mathfrak{a}} \backslash \Gamma}\left(\operatorname{Im}\left(\sigma_{\mathfrak{a}}{ }^{-1} \gamma z\right)^{\varepsilon}+\operatorname{Im}\left(\sigma_{\mathfrak{a}}{ }^{-1} \gamma z\right)^{-\varepsilon}+\operatorname{Im}\left(\sigma_{\mathfrak{a}}{ }^{-1} z\right)^{\varepsilon}\right. \\
& \left.+\operatorname{Im}\left(\sigma_{\mathfrak{a}}{ }^{-1} z\right)^{-\varepsilon}+1\right) \times\left|j\left(\sigma_{\mathfrak{a}}{ }^{-1} \gamma, z\right)\right|^{-k} \\
= & y^{-k / 2} \sum_{\gamma \in \Gamma_{\mathfrak{a}} \backslash \Gamma}\left(\operatorname{Im}\left(\sigma_{\mathfrak{a}}{ }^{-1} \gamma z\right)^{k / 2+\varepsilon}+\operatorname{Im}\left(\sigma_{\mathfrak{a}}{ }^{-1} \gamma z\right)^{k / 2-\varepsilon}\right) \\
& +y^{-k / 2}\left(\operatorname{Im}\left(\sigma_{\mathfrak{a}}{ }^{-1} z\right)^{\varepsilon}+\operatorname{Im}\left(\sigma_{\mathfrak{a}}{ }^{-1} z\right)^{-\varepsilon}+1\right) \sum_{\gamma \in \Gamma_{\mathfrak{a}} \backslash \Gamma} \operatorname{Im}\left(\sigma_{\mathfrak{a}}{ }^{-1} \gamma z\right)^{k / 2}
\end{aligned}
$$

for any $\varepsilon>0$. (The implied constant depends on $\varepsilon$.) Now the usual non-holomorphic Eisenstein series

$$
E_{\mathfrak{a}}(z, s)=\sum_{\gamma \in \Gamma_{\mathfrak{a}} \backslash \Gamma} \operatorname{Im}\left(\sigma_{\mathfrak{a}}^{-1} \gamma z\right)^{s}
$$

is known to be absolutely convergent for $s$ with $\operatorname{Re}(s)>1$ (and uniformly convergent for $s$ in compact sets there); see [I1, Chapter 3] and [Sa, Section 1.4]. Moreover it has the Fourier expansion at the cusp $\mathfrak{b}$,

$$
\begin{aligned}
E_{\mathfrak{a}}\left(\sigma_{\mathfrak{b}} z, s\right) & =\delta_{\mathfrak{a} \mathfrak{b}} y^{s}+\phi_{\mathfrak{a} \mathfrak{b}}(s) y^{1-s}+\sum_{m \neq 0} \phi_{\mathfrak{a} \mathfrak{b}}(m, s) W_{s}(m z) \\
& =\delta_{\mathfrak{a} \mathfrak{b}} y^{s}+\phi_{\mathfrak{a} \mathfrak{b}}(s) y^{1-s}+O\left(e^{-2 \pi y}\right)
\end{aligned}
$$


as $y \rightarrow \infty$ with an implied constant depending only on $s$ and $\Gamma$. This is [1, (6.20)] and is in fact valid for all $s$ in $\mathbb{C}$. The Whittaker function $W_{s}(z)$ is described in [11, (1.26)].

By comparing (4.4) with (4.5) we require $k / 2-\varepsilon>1$ and hence $k>2$ for the absolute and uniform convergence of $P_{\mathfrak{a} m}(z, L)_{k}$. Therefore, for $k \geq 4$, the series $P_{\mathfrak{a} m}(z, L)_{k}$ are absolutely and uniformly convergent (for $z$ in compact sets in $\mathfrak{H}$, say) and satisfy $\mathbf{H}$.

We also easily have

$$
P_{\mathfrak{a} m}(\gamma z, L)_{k} j(\gamma, z)^{-k}=P_{\mathfrak{a} m}(z, L)_{k}+L\left(\gamma^{-1}\right) P_{\mathfrak{a} m}(z)_{k}
$$

which implies that $P_{\mathfrak{a} m}(z, L)_{k}$ satisfies $\mathbf{A}\left(M_{k}(\Gamma)\right)$ for $m=0$ and $\mathbf{A}\left(S_{k}(\Gamma)\right)$ for $m>0$.

Finally, we verify that our functions satisfy the cuspidal growth condition $\mathbf{C}$ by considering the Fourier expansion of these series at $\mathfrak{b}$. Since we have established $\mathbf{H}$ we must have

$$
j\left(\sigma_{\mathfrak{b}}, z\right)^{-k} P_{\mathfrak{a} m}\left(\sigma_{\mathfrak{b}} z, L\right)_{k}=\sum_{n \in \mathbb{Z}} a_{\mathfrak{a} \mathfrak{b}}(n) e(n z)
$$

for some constants $a_{\mathfrak{a} \mathfrak{b}}(n)$. Also, noting that $L(I)=0$ for $I$ the identity element of $\Gamma$ and taking into account $(4.7)$, we have

$$
\begin{aligned}
j\left(\sigma_{\mathfrak{b}}, z\right)^{-k} P_{\mathfrak{a} m}\left(\sigma_{\mathfrak{b}} z, L\right)_{k} & =\sum_{\gamma \in \Gamma_{\mathfrak{a}} \backslash \Gamma} L(\gamma) j\left(\sigma_{\mathfrak{a}}{ }^{-1} \gamma \sigma_{\mathfrak{b}}, z\right)^{-k} e\left(m \sigma_{\mathfrak{a}}{ }^{-1} \gamma \sigma_{\mathfrak{b}} z\right) \\
& \ll y^{-k / 2} \sum_{\gamma \in \Gamma_{\mathfrak{a}} \backslash \Gamma, \gamma \neq \Gamma_{\mathfrak{a}}} L(\gamma) \operatorname{Im}\left(\sigma_{\mathfrak{a}}{ }^{-1} \gamma \sigma_{\mathfrak{b}} z\right)^{k / 2} \\
& \ll y^{-k / 2}\left|E_{\mathfrak{a}}\left(\sigma_{\mathfrak{b}} z, k / 2-\epsilon\right)-\delta_{\mathfrak{a} \mathfrak{b}} y^{k / 2-\epsilon}\right| \\
& \ll\left|\phi_{\mathfrak{a} \mathfrak{b}}(k / 2-\epsilon)\right| y^{1-k+\epsilon} .
\end{aligned}
$$

Therefore, for $m \geqslant 0$, we find that $a_{\mathfrak{a} \mathfrak{b}}(n)=0$ for $n \leqslant 0$. Consequently $P_{\mathfrak{a} m}(z, L)_{k}$ satisfies the cuspidal growth condition $\mathbf{C}$. This is perhaps surprising in the case where $m=0$. This means that on the fundamental domain $\mathfrak{F}$ (corresponding to $\sigma_{\mathfrak{a}}, \sigma_{\mathfrak{b}}$, etc. $), P_{\mathfrak{a} 0}(z, L)_{k}$ has exponential decay at its cusps. This will not be the case on any translates $\gamma \mathfrak{F}$ of $\mathfrak{F}$ with $L(\gamma) \neq 0$ by the automorphy condition.

Theorem 4.3. For $4 \leqslant k \in 2 \mathbb{Z}$ and $g$ the genus of $\Gamma \backslash \mathfrak{H}$, we have

$$
\begin{aligned}
\operatorname{dim} S_{k}^{2}(\Gamma) & =(2 g+1) \operatorname{dim} S_{k}(\Gamma), \\
\operatorname{dim} M_{k}^{2}(\Gamma) & =(2 g+1) \operatorname{dim} M_{k}(\Gamma) .
\end{aligned}
$$

Proof. Note that, for a fixed cusp $\mathfrak{a}$, the Poincaré series $P_{\mathfrak{a} j}\left(z, L_{i}\right)$ are all linearly independent, by (4.8), as $j>0$ runs over integers yielding a basis $P_{\mathfrak{a} j}(z)$ for $S_{k}(\Gamma)$ and as $i$ runs over integers in $\{1, \ldots, 2 g\}$ yielding a basis $L_{i}$ of $\operatorname{Hom}_{0}(\Gamma, \mathbb{C})$. These series are also linearly independent of $S_{k}(\Gamma)$. In this way we obtain (4.9).

A similar argument, using the fact that $P_{\mathfrak{a} 0}(z)$ with $\mathfrak{a}$ running over the inequivalent cusps of $\Gamma \backslash \mathfrak{H}$ form a basis for $E_{k}(\Gamma)$, yields (4.10).

This result also clarifies the direct sums (3.10) and (3.11). For example, a secondorder cusp form is in the space $S_{k}^{2}(\Gamma)^{+}$if and only if it is a linear combination of Poincaré series $P_{\mathfrak{a} m}(z, L)$ with $L$ in the subspace of $\operatorname{Hom}_{0}(\Gamma, \mathbb{C})$ generated by the modular symbols (3.2). The space $S_{k}^{2}(\Gamma)^{-}$consists of linear combinations of Poincaré series $P_{\mathfrak{a} m}(z, \bar{L})$, with the conjugates of the modular symbols. 


\section{Calculating $\operatorname{dim} S_{2}^{2}(\Gamma)$}

We first recall some definitions and terminology and put in place the general framework used in $\mathrm{CO}$, $\mathrm{JO}$. We will then specialize to the objects we need.

For $f_{1}, f_{2}$ in $S_{k}(\Gamma)$, the Petersson inner product is defined by

$$
\left\langle f_{1}, f_{2}\right\rangle=\int_{\Gamma \backslash \mathfrak{H}} f_{1}(z) \overline{f_{2}(z)} y^{k} d \mu z
$$

The weight, $k$, of the inner product should be clear from the context; in the rest of this section they are weight 2, whereas in Section 8 they are all weight 0. (Also recall that we are using this notation for the modular symbols (3.2).)

Following [11, (2.42)] we recall the useful notation

$$
y_{\Gamma}(z)=\max _{\mathfrak{a}}\left(\max _{\gamma \in \Gamma}\left(\operatorname{Im}\left(\sigma_{\mathfrak{a}}^{-1} \gamma z\right)\right)\right),
$$

which measures how close $z \in \mathfrak{H}$ is to a cusp. If $\psi($ or $|\psi|)$ is smooth with weight 0 then it is more convenient to write

$$
\psi(z) \ll y_{\Gamma}(z)^{A}
$$

for example, instead of $\psi\left(\sigma_{\mathfrak{a}} z\right) \ll y^{A}$ for each cusp $\mathfrak{a}$ as $y \rightarrow \infty$. For questions involving the convergence of inner products, it is the growth in these cuspidal zones that is important. For weight 0 second-order forms it makes more sense to consider their growth only inside the fundamental domain $\mathfrak{F}$. We use the notation

$$
y_{\mathfrak{F}}(z)=\max _{\mathfrak{a}}\left(\operatorname{Im}\left({\sigma_{\mathfrak{a}}}^{-1} z\right)\right)
$$

for $z \in \mathfrak{F}$.

Next, we recall from Section 2 the non-holomorphic, weight $k$ Poincaré series

$$
U_{\mathfrak{a} m}(z, s, k)=\sum_{\gamma \in \Gamma_{\mathfrak{a}} \backslash \Gamma} \operatorname{Im}\left({\sigma_{\mathfrak{a}}}^{-1} \gamma z\right)^{s} e\left(m \sigma_{\mathfrak{a}}{ }^{-1} \gamma z\right) \varepsilon\left({\sigma_{\mathfrak{a}}}^{-1} \gamma, z\right)^{-k},
$$

where $\varepsilon(\gamma, z)$ is the 'non-holomorphic' weight factor $j(\gamma, z) /|j(\gamma, z)|$. These series were first studied by Selberg. For simplicity, put $U_{\mathfrak{a} m}(z, s)=U_{\mathfrak{a} m}(z, s, 0)$. The main facts we need for this function are that it converges to an analytic function of $s$ for $\operatorname{Re}(s)>1$ and has a continuation to a neighborhood of $s=1$. These results are contained in Propositions A, B and C below.

Now, for $f(z)$ in $S_{2}(\Gamma)$ we shall require its derivatives and antiderivatives. Define $I_{\mathfrak{a} n}$ to be the $n$th antiderivative of $f$. Precisely, for $n \geqslant 1$, we set

$$
I_{\mathfrak{a} n}\left(z_{n}\right)=\int_{i \infty}^{z_{n}} \cdots \int_{i \infty}^{z_{2}} \int_{i \infty}^{z_{1}} f_{\mathfrak{a}}\left(z_{0}\right) d z_{0} d z_{1} \cdots d z_{n-1}
$$

for $f_{\mathfrak{a}}(z)=f\left(\sigma_{\mathfrak{a}} z\right) / j\left(\sigma_{\mathfrak{a}}, z\right)^{2}$. Thus $\frac{d}{d z} I_{\mathfrak{a} n}(z)=I_{\mathfrak{a}(n-1)}(z)$, and we use this to extend the definition of $I_{\mathfrak{a} n}$ to all $n$ in $\mathbb{Z}$. In particular it may be checked that

$$
\begin{aligned}
& I_{\mathfrak{a} 1}\left(\sigma_{\mathfrak{a}}{ }^{-1} z\right)=F_{\mathfrak{a}}(z):=\int_{\mathfrak{a}}^{z} f(w) d w \\
& I_{\mathfrak{a} 0}\left({\sigma_{\mathfrak{a}}}^{-1} z\right)=f(z) j\left({\sigma_{\mathfrak{a}}}^{-1}, z\right)^{2} .
\end{aligned}
$$


There are two interesting families of series:

$$
\begin{aligned}
& Q_{\mathfrak{a} m}(z, s, n ; f)=\sum_{\gamma \in \Gamma_{\mathfrak{a}} \backslash \Gamma} I_{\mathfrak{a} n}\left(\sigma_{\mathfrak{a}}{ }^{-1} \gamma z\right) \operatorname{Im}\left(\sigma_{\mathfrak{a}}{ }^{-1} \gamma z\right)^{s} e\left(m \sigma_{\mathfrak{a}}{ }^{-1} \gamma z\right), \\
& Q_{\mathfrak{a} m}(z, s, n ; \bar{f})=\sum_{\gamma \in \Gamma_{\mathfrak{a}} \backslash \Gamma} \overline{I_{\mathfrak{a} n}\left(\sigma_{\mathfrak{a}}{ }^{-1} \gamma z\right)} \operatorname{Im}\left(\sigma_{\mathfrak{a}}{ }^{-1} \gamma z\right)^{s} e\left(m \sigma_{\mathfrak{a}}{ }^{-1} \gamma z\right) .
\end{aligned}
$$

For $m=0$ the theory of the series $Q_{\mathfrak{a} m}(z, s, n ; f)$ for all $n$ and $f$ with weight $k \geqslant 2$ is given in [CO]. In [JO, the series $Q_{\mathfrak{a} m}(z, s, 1 ; f)$ with $m \neq 0$ is used to find the analogue of the Kronecker limit formula for second-order, non-holomorphic Eisenstein series. Here we will only need results about $Q_{\mathfrak{a} m}(z, s, n ; \bar{f})$ and only for $n \leqslant 1$.

The next proposition provides the basic convergence results and growth estimates for $U_{\mathfrak{a} m}(z, s, k), Q_{\mathfrak{a} m}(z, s, 1 ; \bar{f})$, and $Q_{\mathfrak{a} m}^{\prime}(z, s, 1 ; \bar{f})$, which is the termwise derivative of (5.2) with respect to $z$, and the weight 2 function

$$
G_{\mathfrak{a} m}(z, s ; \bar{F})=\sum_{\gamma \in \Gamma_{\mathfrak{a}} \backslash \Gamma} \frac{\overline{F_{\mathfrak{a}}(\gamma z)}}{j\left(\sigma_{\mathfrak{a}}{ }^{-1} \gamma, z\right)^{2}} \operatorname{Im}\left(\sigma_{\mathfrak{a}}{ }^{-1} \gamma z\right)^{s} e\left(m \sigma_{\mathfrak{a}}{ }^{-1} \gamma z\right) .
$$

Proposition A. For $k \in 2 \mathbb{Z}$ and $\sigma=\operatorname{Re}(s)>1$, the series $U_{\mathfrak{a} m}(z, s, k)$, $Q_{\mathfrak{a} m}(z, s, 1 ; \bar{f}), Q_{\mathfrak{a} m}^{\prime}(z, s, 1 ; \bar{f})$ and $G_{\mathfrak{a} m}(z, s-1 ; \bar{F})$ converge absolutely and uniformly on compact sets to analytic functions of $s$. For these $s$ we have

$$
\begin{aligned}
U_{\mathfrak{a} 0}(z, s, k) & \ll y_{\Gamma}(z)^{\sigma}, \\
U_{\mathfrak{a} m}(z, s, k) & \ll 1, \quad m>0, \\
Q_{\mathfrak{a} m}(z, s, 1 ; \bar{f}) & \ll y_{\Gamma}(z)^{1 / 2-\sigma / 2}, \quad m \geqslant 0, \\
y Q_{\mathfrak{a} m}^{\prime}(z, s, 1 ; \bar{f}) & \ll(|m|+1) y_{\Gamma}(z)^{1 / 2-\sigma / 2}, \quad m \geqslant 0, \\
y G_{\mathfrak{a} m}(z, s-1 ; \bar{F}) & \ll y_{\Gamma}(z)^{1 / 2-\sigma / 2}, \quad m \geqslant 0,
\end{aligned}
$$

where the implied constants depend on $s, k, f$ and $\Gamma$ but not on $m$.

The proof of this proposition is not difficult and essentially amounts to comparing these series with the standard Eisenstein series $E_{\mathfrak{a}}(z, s)$. We give the details in Section 9. The next propositions show the analytic continuation of the series $U_{\mathfrak{a} m}$, $Q_{\mathfrak{a} m}$ and $Q_{\mathfrak{a} m}^{\prime}$ to a neighborhood of $s=1$. This is essential for our construction of weight two second-order forms. Again, to keep the flow of ideas intact, we relegate the proofs to Sections 10 and 11.

First we choose, once and for all, a constant $\delta_{\Gamma}$ depending on $\Gamma$ with $0<\delta_{\Gamma}<$ $1 / 2$. It is chosen so that poles appearing from the discrete spectrum have real part less than $1-\delta_{\Gamma}$. See the discussion at the beginning of Section 8 for a complete explanation.

Proposition B. For $k \in 2 \mathbb{Z}$ the (Eisenstein) series $U_{\mathfrak{a} 0}(z, s, k)$ has a meromorphic continuation to all $s$ with $\operatorname{Re}(s)>1-\delta_{\Gamma}$. We have

$$
U_{\mathfrak{a} 0}(z, s, k) \ll y_{\Gamma}(z)^{\sigma}
$$

for these $s$ with the implied constant depending on $s, k$ and $\Gamma$. The only possible pole in this region appears at $s=1$ when $k=0$. It is a simple pole with residue $1 / V$. 
Proposition C. For $k \in 2 \mathbb{Z}$ and $m>0$, the Poincaré series $U_{\mathfrak{a} m}(z, s, k)$ has a continuation to an analytic function for all $s$ with $\operatorname{Re}(s)>1-\delta_{\Gamma}$. We have

$$
U_{\mathfrak{a} m}(z, s, k) \ll y_{\Gamma}(z)^{1 / 2}
$$

for these $s$ with the implied constant depending on $s, m, k$ and $\Gamma$.

This proposition should be standard and the proof of the first part can be found in $[\mathrm{Se}$. However, since we have not found a reference for the growth estimate, we provide a proof in Section 10 based on the methods of [JO, Section 8].

Proposition D. For $m \geqslant 0$, both series $(s-1) Q_{\mathfrak{a} m}(z, s, 1 ; \bar{f})$ and $Q_{\mathfrak{a} m}^{\prime}(z, s, 1 ; \bar{f})$ have continuations to analytic functions of $s$ with $\operatorname{Re}(s)>1-\delta_{\Gamma}$. For these $s$ values,

$$
(s-1) Q_{\mathfrak{a} m}(z, s, 1 ; \bar{f}), \quad y Q_{\mathfrak{a} m}^{\prime}(z, s, 1 ; \bar{f}) \ll y_{\Gamma}(z)^{1 / 2} .
$$

The implied constant depends on $s, m, f$ and $\Gamma$. Also $Q_{\mathfrak{a} m}(z, s, 1 ; \bar{f})$ has a simple pole at $s=1$ with residue $2 i \overline{\left\langle f, P_{\mathfrak{a} m}(\cdot)_{2}\right\rangle}$.

The $P_{\mathfrak{a} m}(z)_{2}$ appearing in the above residue is

$$
P_{\mathfrak{a} m}(z)_{2}=y^{-1} U_{\mathfrak{a} m}(z, 1,2),
$$

which is well defined thanks to Propositions B and C. These series are holomorphic for $m>0$ and span $S_{2}(\Gamma)$; see [JO, Theorem 3.2] for example. For $m=0$ they are no longer holomorphic. One way to see this is to note that

$$
P_{\mathfrak{a} 0}(z)_{2}=y^{-1} U_{\mathfrak{a} 0}(z, 1,2)=y^{-1} \lim _{s \rightarrow 1} R_{0} E_{\mathfrak{a}}(z, s)=2 i \lim _{s \rightarrow 1} \frac{d}{d z} E_{\mathfrak{a}}(z, s),
$$

which by (4.7) satisfies

$$
j\left(\sigma_{\mathfrak{b}}, z\right)^{-2} P_{\mathfrak{a} 0}\left(\sigma_{\mathfrak{b}} z\right)_{2}=\delta_{\mathfrak{a} \mathfrak{b}}-\frac{1}{y V}+O\left(e^{-2 \pi y}\right)
$$

as $y \rightarrow \infty$ and

$$
y^{2} \frac{d}{d \bar{z}} P_{\mathfrak{a} 0}(z)_{2}=\frac{i}{2 V} .
$$

These functions were also used in $\mathrm{GO}$, where it is shown that for any two distinct cusps $\mathfrak{a}$ and $\mathfrak{b}$, the differences $P_{\mathfrak{a} 0}(z)_{2}-P_{\mathfrak{b} 0}(z)_{2}$ span $E_{2}(\Gamma)$, the space of Eisenstein series (4.2). The operator $R_{0}=2 i y d / d z$ is the weight raising operator as discussed in Section 8.

Remark. In reality all the series we are considering here, $U_{\mathfrak{a} m}(z, s, k), Q_{\mathfrak{a} m}(z, s, 1 ; \bar{f})$, $y Q_{\mathfrak{a} m}^{\prime}(z, s, 1 ; \bar{f})$ and $y G_{\mathfrak{a} m}(z, s-1 ; \bar{F})$, have meromorphic continuations to all $s$ in $\mathbb{C}$ and indeed it is a relatively straightforward exercise to extend the proofs of Propositions $\mathrm{C}$ and $\mathrm{D}$ to the entire $s$ plane. The reason we restrict our attention to $\operatorname{Re}(s)>1-\delta_{\Gamma}$ is to avoid having to include details concerning poles coming from the exceptional spectrum and on the line $\operatorname{Re}(s)=1 / 2$. For our purposes we only require continuation to a neighborhood of $s=1$. See [CO] for details of how these techniques extend to all of $\mathbb{C}$.

We are now ready to turn to the dimension of $S_{2}^{2}(\Gamma)$. Because of the decomposition (3.7), in order to compute it, we need to investigate the space $S_{2}^{2}(\Gamma)^{-}$. In view of the remarks following Theorem 4.3, we would like to construct the series

$$
\text { " } P_{\mathfrak{a} m}(z, L)_{2} "=\sum_{\gamma \in \Gamma_{\mathfrak{a}} \backslash \Gamma} L(\gamma) j\left({\sigma_{\mathfrak{a}}}^{-1} \gamma, z\right)^{-2} e\left(m{\sigma_{\mathfrak{a}}}^{-1} \gamma z\right)
$$


with $L(\gamma)=\overline{\langle\gamma, f\rangle}, f \in S_{2}(\Gamma)$. Unfortunately this series is not absolutely convergent. However, if it were, it would formally equal

$$
\begin{aligned}
\sum_{\gamma \in \Gamma_{\mathfrak{a}} \backslash \Gamma} \overline{\left(F_{\mathfrak{a}}(\gamma z)-F_{\mathfrak{a}}(z)\right)} j\left(\sigma_{\mathfrak{a}}{ }^{-1} \gamma, z\right)^{-2} \operatorname{Im}\left(\sigma_{\mathfrak{a}}{ }^{-1} \gamma z\right)^{s} e\left(m \sigma_{\mathfrak{a}}{ }^{-1} \gamma z\right) \\
=G_{\mathfrak{a} m}(z, s ; \bar{F})-\overline{F_{\mathfrak{a}}(z)} y^{-1} U_{\mathfrak{a} m}(z, s+1,2)
\end{aligned}
$$

with $s=0$. This motivates us to study

$$
\begin{aligned}
Z_{\mathfrak{a} m}(z, s ; f) & =\sum_{\gamma \in \Gamma_{\mathfrak{a}} \backslash \Gamma} \overline{\langle\gamma, f\rangle} \frac{\operatorname{Im}\left(\sigma_{\mathfrak{a}}{ }^{-1} \gamma z\right)^{s}}{j\left(\sigma_{\mathfrak{a}}{ }^{-1} \gamma, z\right)^{2}} e\left(m \sigma_{\mathfrak{a}}{ }^{-1} \gamma z\right) \\
& =G_{\mathfrak{a} m}(z, s ; \bar{F})-\overline{F_{\mathfrak{a}}(z)} y^{-1} U_{\mathfrak{a} m}(z, s+1,2)
\end{aligned}
$$

and show that it has an analytic continuation to $s=0$. An analysis will show that it does and has the correct growth at cusps but is not always analytic. Nonetheless, this construction provides us with all remaining elements of $S_{2}^{2}(\Gamma)$ and $M_{2}^{2}(\Gamma)$. The next proposition develops the required properties of $Z_{\mathfrak{a} m}$.

Proposition 5.1. Let $m$ be an integer $\geqslant 0, f \in S_{2}(\Gamma)$ and $\mathfrak{a}$ a cusp. Then $Z_{\mathfrak{a} m}(z, s ; f)$ as defined in (5.7) admits an analytic continuation to $\operatorname{Re}(s)>-\delta_{\Gamma}$. Also

$$
\begin{aligned}
Z_{\mathfrak{a} m}(\gamma z, 0 ; f) j(\gamma, z)^{-2} & =Z_{\mathfrak{a} m}(z, 0 ; f)-\overline{\langle\gamma, f\rangle} P_{\mathfrak{a} m}(z)_{2}, \\
y Z_{\mathfrak{a} m}(z, 0 ; f) & \ll y_{\mathfrak{F}}(z)^{1 / 2} \\
\frac{d}{d \bar{z}} Z_{\mathfrak{a} m}(z, 0 ; f) & =-y^{-2} \overline{\left\langle f, P_{\mathfrak{a} m}(\cdot)_{2}\right\rangle}+\delta_{m, 0} \overline{F_{\mathfrak{a}}(z)} /\left(2 i y^{2} V\right),
\end{aligned}
$$

where (i) is true for all $\gamma$ in $\Gamma$ and the implied constant in (ii) is independent of $z$.

Proof. We have already seen in Proposition A, part (v) that $G_{\mathfrak{a} m}(z, s ; \bar{F})$ is absolutely convergent for $\sigma=\operatorname{Re}(s)>0$ and satisfies

$$
y G_{\mathfrak{a} m}(z, s ; \bar{F}) \ll y_{\Gamma}(z)^{-\sigma / 2}
$$

for these $s$ and an implied constant independent of $z$. Now use the relation

$$
\frac{d}{d z} Q_{\mathfrak{a} m}(z, s, 1 ; \bar{f})=\frac{-i s}{2} G_{\mathfrak{a} m}(z, s-1 ; \bar{F})+2 \pi i m G_{\mathfrak{a} m}(z, s ; \bar{F})
$$

to see that

$$
G_{\mathfrak{a} m}(z, s ; \bar{F})=\frac{4 \pi m}{s+1} G_{\mathfrak{a} m}(z, s+1 ; \bar{F})+\frac{2 i}{s+1} Q_{\mathfrak{a} m}^{\prime}(z, s+1,1 ; \bar{f}) .
$$

With Proposition D this gives the analytic continuation of $G_{\mathfrak{a} m}(z, s ; \bar{F})$ to $\operatorname{Re}(s)>$ $-\delta_{\Gamma}$. Together with Propositions B and C, we see that both terms on the right of (5.7) have analytic continuations to $\operatorname{Re}(s)>-\delta_{\Gamma}$, proving the first statement of the proposition.

Equations (5.7) and (5.9) now imply that

$$
y Z_{\mathfrak{a} m}(z, 0 ; f)=4 \pi m y G_{\mathfrak{a} m}(z, 1 ; \bar{F})+2 i y Q_{\mathfrak{a} m}^{\prime}(z, 1,1 ; \bar{f})-\overline{F_{\mathfrak{a}}(z)} U_{\mathfrak{a} m}(z, 1,2) .
$$

With Proposition A, part (v) and Proposition D we have

$$
y G_{\mathfrak{a} m}(z, 1 ; \bar{F}) \ll y_{\Gamma}(z)^{-1 / 2}, \quad y Q_{\mathfrak{a} m}^{\prime}(z, 1,1 ; \bar{f}) \ll y_{\Gamma}(z)^{1 / 2} .
$$


To bound $\overline{F_{\mathfrak{a}}(z)} U_{\mathfrak{a} m}(z, 1,2)$ we first note that for $m \neq 0$ we have $U_{\mathfrak{a} m}(z, 1,2) \ll$ $y_{\Gamma}(z)^{1 / 2}$ by Proposition $\mathrm{C}$ and for $m=0$ we have $U_{\mathfrak{a} m}\left(\sigma_{\mathfrak{b}} z, 1,2\right) \ll \delta_{\mathfrak{a} \mathfrak{b}} y+1$ as $y \rightarrow \infty$ by (10.3). Also it is easy to show that, for $z$ in $\mathfrak{F}$,

$$
F_{\mathfrak{a}}\left(\sigma_{\mathfrak{a}} z\right) \ll e^{-2 \pi y}, \quad F_{\mathfrak{a}}\left(\sigma_{\mathfrak{b}} z\right) \ll 1, \mathfrak{a} \neq \mathfrak{b}
$$

as $y \rightarrow \infty$ (see (9.3) and (9.4)). Part (ii) of the proposition now follows. It is also easy to check that, for $\operatorname{Re}(s)$ large,

$$
Z_{\mathfrak{a} m}(\gamma z, s ; f) j(\gamma, z)^{-2}=Z_{\mathfrak{a} m}(z, s ; f)-\overline{\langle\gamma, f\rangle} y^{-1} U_{\mathfrak{a} m}(z, s+1,2),
$$

thus deducing (i) by analytic continuation. A lengthy but routine calculation yields, again for $\operatorname{Re}(s)$ large,

$$
\frac{d}{d \bar{z}} Z_{\mathfrak{a} m}(z, s ; f)=\frac{i s}{2 y^{2}}\left(Q_{\mathfrak{a} m}(z, s+1,1 ; \bar{f})-\overline{F_{\mathfrak{a}}(z)} U_{\mathfrak{a} m}(z, s+1)\right) .
$$

Combined with Propositions B, C and D we then find that (iii) holds. This completes all the parts of Proposition 5.1.

Before we continue we define a new function, $Z_{\lambda, \mu}$, that is more convenient to work with than $Z_{\mathfrak{a} m}$. As previously noted, for different values of $m>0, P_{\mathfrak{a} m}(\cdot)_{2}$ spans $S_{2}(\Gamma)$. So for any pair $(\lambda, \mu)$ of elements in $S_{2}(\Gamma)$ we may find a linear combination $Z_{\lambda, \mu}$ of $Z_{\mathfrak{a} m}(z, 0 ; \lambda)$ with $m>0$ such that, for all $\gamma$ in $\Gamma$,

$$
\begin{aligned}
\left.Z_{\lambda, \mu}\right|_{2}(\gamma-1) & =\overline{\langle\gamma, \lambda\rangle} \mu, \\
y^{2} \frac{d}{d \bar{z}} Z_{\lambda, \mu} & =\overline{\langle\lambda, \mu\rangle}, \\
y Z_{\lambda, \mu} & \ll y_{\mathfrak{F}}(z)^{1 / 2} .
\end{aligned}
$$

To prove that the dimension of $S_{2}^{2}(\Gamma)$ is one less than we would initially expect, we will also need the next result.

Proposition 5.2. For any $\mu \neq 0$ in $S_{2}(\Gamma)$ it is impossible for an element $f$ of $S_{2}^{2}(\Gamma)$ to satisfy

$$
\left.f\right|_{2}(\gamma-1)=\overline{\langle\gamma, \mu\rangle} \mu
$$

for all $\gamma$ in $\Gamma$.

Proof. The main idea is to use $P_{\mathfrak{a} 0}(z)_{2} \in \tilde{M}_{2}(\Gamma)$ and consider the sum $Z_{\mu, \mu}+$ $2 i V \overline{\langle\mu, \mu\rangle} P_{\mathfrak{a} 0}(\cdot)_{2}$. Combine (5.5) and (5.11) to obtain

$$
\frac{d}{d \bar{z}}\left(Z_{\mu, \mu}(z)+2 i V \overline{\langle\mu, \mu\rangle} P_{\mathfrak{a} 0}(z)_{2}\right)=0 .
$$

Looking at each cusp we see by (5.4) and (5.12) that

$$
j\left(\sigma_{\mathfrak{b}}, z\right)^{-2}\left(Z_{\mu, \mu}\left(\sigma_{\mathfrak{b}} z\right)+2 i V \overline{\langle\mu, \mu\rangle} P_{\mathfrak{a} 0}\left(\sigma_{\mathfrak{b}} z\right)_{2}\right) \ll y^{-1 / 2}+\delta_{\mathfrak{a} \mathfrak{b}}+\frac{1}{y V}
$$

as $y \rightarrow \infty$, so the Fourier expansion of $Z_{\mu, \mu}(z)+2 i V \overline{\langle\mu, \mu\rangle} P_{\mathfrak{a} 0}(z)_{2}$ has only nonnegative terms and only a constant term at the cusp a. Now suppose $f$, as described in the proposition, does exist. Then

$$
\lambda=f-Z_{\mu, \mu}-2 i V \overline{\langle\mu, \mu\rangle} P_{\mathfrak{a} 0}(\cdot)_{2}
$$

is an element of $M_{2}(\Gamma)$ that has exponential decay at every cusp except possibly one by the previous argument. But $P_{\mathfrak{a} 0}(\cdot)_{2}-P_{\mathfrak{b} 0}(\cdot)_{2}$ span $E_{2}(\Gamma)$, and hence every 
element of $E_{2}(\Gamma)$ must not have exponential decay at at least two distinct cusps. It follows that $\lambda$ must be in $S_{2}(\Gamma)$. This yields a contradiction on writing

$$
\lambda(z)-f(z)+Z_{\mu, \mu}(z)=-2 i V \overline{\langle\mu, \mu\rangle} P_{\mathfrak{a} 0}(z)_{2}
$$

and examining the size of both sides as $z \rightarrow \mathfrak{a}$.

Theorem 5.3. Let $\left\{f_{1}, \ldots, f_{g}\right\}$ be an orthonormal basis of $S_{2}(\Gamma)$. Then the set

$$
A=\left\{Z_{f_{i}, f_{j}}\right\}_{i \neq j} \cup\left\{Z_{f_{i}, f_{i}}-Z_{f_{1}, f_{1}}\right\}_{i=2}^{g} \cup\left\{f_{i} \int f_{j}\right\}_{i, j} \cup\left\{f_{i}\right\}_{i}
$$

is a basis of $S_{2}^{2}(\Gamma)$.

Proof. We first observe that, with (5.10), (5.11) and (5.12), all functions belong to $S_{2}^{2}(\Gamma)$. Next we show that $A$ generates $S_{2}^{2}(\Gamma)$. Equations (3.3) and (3.5) imply that if $f \in S_{2}^{2}(\Gamma)$, then there are $\lambda_{i j}, \mu_{i j} \in \mathbb{C}$ such that

$$
\left.f\right|_{2}(\gamma-1)=\sum_{i, j}\left(\lambda_{i j}\left\langle\gamma, f_{i}\right\rangle+\mu_{i j} \overline{\left\langle\gamma, f_{i}\right\rangle}\right) f_{j}
$$

for all $\gamma$ in $\Gamma$. Set

$$
f^{*}=f-\sum_{i, j} \lambda_{i j} f_{j} \int f_{i}-\sum_{i, j} \mu_{i j} Z_{f_{i}, f_{j}} .
$$

Thanks to (5.10), $\left.f^{*}\right|_{2}(\gamma-1)=0$ for all $\gamma$ in $\Gamma$. Writing

$f^{*}=f-\sum_{i, j} \lambda_{i j} f_{j} \int f_{i}-\sum_{i \neq j} \mu_{i j} Z_{f_{i}, f_{j}}-\sum_{i} \mu_{i i}\left(Z_{f_{i}, f_{i}}-Z_{f_{1}, f_{1}}\right)-\left(\sum_{i} \mu_{i i}\right) Z_{f_{1}, f_{1}}$ makes it clear by (5.11) that

$$
y^{2} \frac{d}{d \bar{z}} f^{*}=-\left(\sum_{i} \mu_{i i}\right) \overline{\left\langle f_{1}, f_{1}\right\rangle} .
$$

Hence $f^{*}+\left(\sum_{i} \mu_{i i}\right) Z_{f_{1}, f_{1}}$ is holomorphic.

With Lemma 4.1, $f_{i} \int_{z_{0}}^{z} \lambda_{i}(w) d w$ satisfies condition C. Also, with (5.12),

$$
j\left(\sigma_{\mathfrak{b}}, z\right)^{-2} Z_{f_{i}, f_{j}}\left(\sigma_{\mathfrak{b}} z\right) \ll \operatorname{Im}\left(\sigma_{\mathfrak{b}} z\right)^{-1}\left|j\left(\sigma_{\mathfrak{b}}, z\right)\right|^{-2} y^{1 / 2}=y^{-1 / 2}
$$

as $y \rightarrow \infty$. Therefore $f^{*}+\left(\sum_{i} \mu_{i i}\right) Z_{f_{1}, f_{1}}$ must have a Fourier expansion at each cusp with only positive terms. In other words,

$$
j\left(\sigma_{\mathfrak{b}}, z\right)^{-2}\left(f^{*}\left(\sigma_{\mathfrak{b}} z\right)+\left(\sum_{i} \mu_{i i}\right) Z_{f_{1}, f_{1}}\left(\sigma_{\mathfrak{b}} z\right)\right)=\sum_{n=1}^{\infty} d_{\mathfrak{b}}(n) e^{2 \pi i n z}
$$

for constants $d_{\mathfrak{b}}(n)$ and hence $f^{*}+\left(\sum_{i} \mu_{i i}\right) Z_{f_{1}, f_{1}}$ satisfies condition $\mathbf{C}$.

Further, if $\sum_{i} \mu_{i i} \neq 0$, then for all $\gamma \in \Gamma$,

$$
\left.\frac{f^{*}+\left(\sum_{i} \mu_{i i}\right) Z_{f_{1}, f_{1}}}{\sum_{i} \mu_{i i}}\right|_{k}(\gamma-1)=\left.Z_{f_{1}, f_{1}}\right|_{k}(\gamma-1)=\overline{\left\langle\gamma, f_{1}\right\rangle} f_{1},
$$

which contradicts Proposition 5.2. Consequently $\sum_{i} \mu_{i i}=0, f^{*} \in S_{2}(\Gamma)$ and $A$ generates $S_{2}^{2}(\Gamma)$. 
Finally, we verify that $A$ is linearly independent. Suppose that for some $k_{i j}, l_{i j}$, $n_{i}, m_{i} \in \mathbb{C}$, we have

$$
\sum_{i \neq j} k_{i j} Z_{f_{i}, f_{j}}+\sum_{i, j} l_{i j} f_{j} \int f_{i}+\sum_{i \neq 1} n_{i}\left(Z_{f_{i}, f_{i}}-Z_{f_{1}, f_{1}}\right)+\sum_{i} m_{i} f_{i}=0 .
$$

With (5.10), if we let $\gamma-1(\gamma \in \Gamma)$ act on both sides, we obtain:

$$
\sum_{i \neq j} k_{i j} \overline{\left\langle\gamma, f_{i}\right\rangle} f_{j}+\sum_{i, j} l_{i j}\left\langle\gamma, f_{i}\right\rangle f_{j}+\sum_{j \neq 0} n_{j}\left(\overline{\left\langle\gamma, f_{j}\right\rangle} f_{j}-\overline{\left\langle\gamma, f_{1}\right\rangle} f_{1}\right)=0 .
$$

Take the inner product of both sides with $f_{j}, j \neq 1$ to see that

$$
\sum_{i \neq j} k_{i j} \overline{\left\langle\gamma, f_{i}\right\rangle}+\sum_{i} l_{i j}\left\langle\gamma, f_{i}\right\rangle+n_{j} \overline{\left\langle\gamma, f_{j}\right\rangle}=0 .
$$

Therefore $k_{i j}, l_{i j}$ and $n_{j}$ are all 0 for $j \neq 1$ by the usual Eichler-Shimura isomorphism and similarly for $j=1$. Hence the constants $m_{i}$ must vanish too.

\section{Corollary 5.4.}

$$
\operatorname{dim} S_{2}^{2}(\Gamma)=\left\{\begin{array}{l}
0 \text { if } \operatorname{dim} S_{2}(\Gamma)=0, \\
(2 g+1) \operatorname{dim} S_{2}(\Gamma)-1 \text { otherwise. }
\end{array}\right.
$$

Remark 5.5. As a byproduct of the proof of Proposition 5.2, we have also shown that the interesting form

$$
Z_{\mu, \mu}+2 i V \overline{\langle\mu, \mu\rangle} P_{\mathfrak{a} 0}(\cdot)_{2}
$$

exists and is in $R_{2}^{2}(\Gamma)$ but not in $S_{2}^{2}(\Gamma)$.

\section{Calculating $\operatorname{dim} M_{k}^{2}(\Gamma)$ FOR $k \leqslant 2$}

First we work on the case $k=2$. For any $f \in S_{2}(\Gamma)$ and cusps $\mathfrak{a}, \mathfrak{b}$ put

$$
\mathcal{Z}_{\mathfrak{a} \mathfrak{b}}(z ; f)=Z_{\mathfrak{a} 0}(z, 0 ; f)-Z_{\mathfrak{b} 0}(z, 0 ; f)+P_{\mathfrak{a} 0}(z)_{2} \overline{\int_{\mathfrak{a}}^{\mathfrak{b}} f(w) d w} .
$$

With Proposition 5.1, part (iii) and (5.5), we have

$$
-y^{2} \frac{d}{d \bar{z}} \mathcal{Z}_{\mathfrak{a} \mathfrak{b}}(z ; f)=\overline{\left\langle f, P_{\mathfrak{a} 0}(\cdot)_{2}-P_{\mathfrak{b} 0}(\cdot)_{2}\right\rangle} .
$$

It is routine to verify by unfolding that $\left\langle f, \operatorname{Im}(\cdot)^{-1} U_{\mathfrak{a} 0}(\cdot, s, 2)\right\rangle=0$ for $\operatorname{Re}(s)$ large. It follows by analytic continuation that $\left\langle f, P_{\mathfrak{a} 0}(\cdot)_{2}\right\rangle=0$ and hence that

$$
\frac{d}{d \bar{z}} \mathcal{Z}_{\mathfrak{a} \mathfrak{b}}(z ; f)=0 \text {. }
$$

So $\mathcal{Z}_{\mathfrak{a} \mathfrak{b}}(z ; f)$ satisfies $\mathbf{H}$ and, with (ii) of Proposition 5.1 and (5.4), it satisfies condition N. By part (i) of the same proposition,

$$
\mathcal{Z}_{\mathfrak{a} \mathfrak{b}}(\gamma z ; f) j(\gamma, z)^{-2}=\mathcal{Z}_{\mathfrak{a} \mathfrak{b}}(z ; f)-\overline{\langle\gamma, f\rangle}\left(P_{\mathfrak{a} 0}(z)_{2}-P_{\mathfrak{b} 0}(z)_{2}\right)
$$

and conditions $\mathbf{A}\left(M_{k}(\Gamma)\right)$ and $\mathbf{P}$ hold. We have shown that $\mathcal{Z}_{\mathfrak{a} \mathfrak{b}}(z ; f) \in M_{2}^{2}(\Gamma)$ for all cusps $\mathfrak{a}, \mathfrak{b}$ and cusp forms $f$. As we already noted, the differences $P_{\mathfrak{a} 0}(z)_{2}-$ $P_{\mathfrak{b} 0}(z)_{2}$ span $E_{2}(\Gamma)$ (and are orthogonal to $S_{2}(\Gamma)$ ). So for any $f \in S_{2}(\Gamma)$ and $h \in E_{2}(\Gamma)$ we may find a linear combination, $Z_{f, h}$, of $\mathcal{Z}_{\mathfrak{a} \mathfrak{b}}(z ; f)$ for different $\mathfrak{a}, \mathfrak{b}$ that is holomorphic and satisfies

$$
\left.Z_{f, h}\right|_{2}(\gamma-1)=\overline{\langle\gamma, f\rangle} h
$$


The next theorem is easy to check using Remark 5.5, and the corollary follows quickly.

Theorem 6.1. Let $\left\{f_{1}, \ldots, f_{g}\right\}$ be an orthonormal basis of $S_{2}(\Gamma)$ and let $\left\{h_{1}, \ldots, h_{p-1}\right\}$ be a basis for $E_{2}(\Gamma)$. If $\mathfrak{a}$ is a fixed cusp, then the set

$\left\{Z_{f_{i}, f_{j}}\right\}_{i \neq j} \cup\left\{Z_{f_{i}, f_{i}}+2 i V P_{\mathfrak{a} 0}(\cdot)_{2}\right\}_{i} \cup\left\{Z_{f_{i}, h_{j}}\right\}_{i, j} \cup\left\{f_{i} \int f_{j}\right\}_{i, j} \cup\left\{h_{j} \int f_{i}\right\}_{i, j} \cup\left\{f_{i}\right\}_{i} \cup\left\{h_{j}\right\}_{j}$ is a basis for $M_{2}^{2}(\Gamma)$.

\section{Corollary 6.2.}

$$
\operatorname{dim} M_{2}^{2}(\Gamma)=(2 g+1) \operatorname{dim} M_{2}(\Gamma) .
$$

Next we look at $M_{0}^{2}(\Gamma)$.

Theorem 6.3. With $\left\{f_{1}, \ldots, f_{g}\right\}$ a basis of $S_{2}(\Gamma)$, a basis for $M_{0}^{2}(\Gamma)$ is

$$
\{1\} \cup\left\{\int f_{i}\right\}_{i}
$$

and $\operatorname{dim} M_{0}^{2}(\Gamma)=g+1$.

Proof. Let $f \in M_{0}^{2}(\Gamma)$. Since $M_{0}(\Gamma)$ consists of the constant functions, for each $\gamma \in \Gamma,\left.f\right|_{0}(\gamma-1)=c_{\gamma} \in \mathbb{C}$. However $f$ belongs to $M_{0}^{2}(\Gamma)$, so the map $\gamma \rightarrow c_{\gamma}$ is a parabolic 1-cocycle in terms of the trivial action of $\Gamma$ on $\mathbb{C}$. Hence, by the EichlerShimura isomorphism, there are $g, h \in S_{2}(\Gamma)$ such that $c_{\gamma}=\langle\gamma, g\rangle+\overline{\langle\gamma, h\rangle}$. This is equivalent to

$$
\left.\left(f(z)-\int_{z_{0}}^{z} g(w) d w\right)\right|_{0}(\gamma-1)=\overline{\langle\gamma, h\rangle} .
$$

Set $F(z)=f(z)-\int_{z_{0}}^{z} g(w) d w$. An easy computation shows that $F^{\prime}=f^{\prime}-g$ has weight 2. Using the Fourier expansion of $f$ at any cusp, we also deduce that $F^{\prime}$ satisfies condition $\mathbf{C}$. Hence $F^{\prime} \in S_{2}(\Gamma)$ and

$$
\left(\left.F\right|_{0}(\gamma-1)\right)(z)=F(\gamma z)-F(z)=\int_{z}^{\gamma z} F^{\prime}(w) d w=\left\langle\gamma, F^{\prime}\right\rangle .
$$

Because of the Eichler-Shimura isomorphism, this together with (6.2) implies that $F^{\prime}=h \equiv 0$. Hence, since $f$ is holomorphic, $f(z)=\int_{z_{0}}^{z} g(w) d w+c$, where $c$ is a constant. This shows that (6.1) spans $M_{0}^{2}(\Gamma)$. Linear independence follows as in the proof of Theorem 5.3.

It is easy to check that when the first-order space is 0 that the corresponding second-order space must be 0 . For even $-k \leqslant-2$ we have $M_{-k}(\Gamma)=0$. Hence any $f \in M_{-k}^{2}(\Gamma)$ satisfies $\left.f\right|_{-k}(\gamma-1)=0$ for all $\gamma$ in $\Gamma$. Therefore $f \in M_{-k}(\Gamma)$ and $f \equiv 0$. The same argument shows that $S_{-k}^{2}(\Gamma)=0$ for all even $-k \leqslant 0$.

All parts of Theorems 2.1 and 2.2 are now complete.

\section{An EICHLER-Shimura-LIKE ISOMORPHISM}

For $k \geqslant 2$ let $P_{k-2}$ denote the space of polynomials of degree at most $k-2$ with coefficients in $\mathbb{C}$. With $F$ in $M_{k}^{2}(\Gamma)$ define a map $\phi: \Gamma \rightarrow P_{k-2}$ by the formula

$$
\phi(\gamma)=\int_{i}^{\gamma^{-1} i} F(z)(z-X)^{k-2} d z
$$


for all $\gamma \in \Gamma$, where $X$ is the polynomial variable and integration takes place on a geodesic in the upper-half plane. This is the exact analogue of the period polynomial map of Eichler cohomology and, as in the classical case, it has a close relation to the values of $L(s, F)$ at $s=1, \ldots, k-1$. Specifically, if $F$ is cuspidal, $i$ can be replaced by $i \infty$ in the definition of $\phi$, and then the polynomial coefficients are linear combinations of values of the additively twisted $L$-function of $F$ which is

$$
\sum_{n=1}^{\infty} \frac{a_{n} e^{2 \pi i x n}}{n^{s}} \text { for } F(z)=\sum_{n=1}^{\infty} a_{n} e^{2 \pi i n z} .
$$

As usual, for every $\mathbb{C}[\Gamma]$-module $M$ we let $d$ denote the coboundary operator on the group of $i$-cochains $C^{i}(\Gamma, M):=\left\{f: \Gamma^{i} \rightarrow M\right\}$. For example, for every $\psi: \Gamma \rightarrow M$,

$$
(d \psi)\left(\gamma_{1}, \gamma_{2}\right)=\psi\left(\gamma_{2}\right) \cdot \gamma_{1}-\psi\left(\gamma_{2} \gamma_{1}\right)+\psi\left(\gamma_{1}\right)
$$

where . denotes the action of $\Gamma$ on $M$. Also, for $\psi: \Gamma^{2} \rightarrow M$,

$$
(d \psi)\left(\gamma_{1}, \gamma_{2}, \gamma_{3}\right)=\psi\left(\gamma_{2}, \gamma_{3}\right) \cdot \gamma_{1}-\psi\left(\gamma_{2} \gamma_{1}, \gamma_{3}\right)+\psi\left(\gamma_{1}, \gamma_{3} \gamma_{2}\right)-\psi\left(\gamma_{1}, \gamma_{2}\right) .
$$

We write $Z^{i}(\Gamma, M), B^{i}(\Gamma, M)$ and $H^{i}(\Gamma, M)$ for the groups of $i$-cocycles, $i$-coboundaries and $i$-cohomology classes respectively. See, for example, [Sh, p. 223] for more details.

We also define the group of parabolic 1-cochains:

$$
C_{p a r}^{1}(\Gamma, M)=\left\{f \in C^{1}(\Gamma, M) \mid f(\pi) \in M .(\pi-1) \text { for all parabolic } \pi \text { in } \Gamma\right\} .
$$

Set $Z_{\text {par }}^{1}(\Gamma, M)=Z^{1}(\Gamma, M) \cap C_{\text {par }}^{1}(\Gamma, M)$ and $B_{\text {par }}^{2}(\Gamma, M)=d\left(C_{\text {par }}^{1}(\Gamma, M)\right)$. With this notation define $H_{\text {par }}^{1}(\Gamma, M)=Z_{\text {par }}^{1}(\Gamma, M) / B^{1}(\Gamma, M)$ and $H_{p a r}^{2}(\Gamma, M)=$ $Z^{2}(\Gamma, M) / B_{\text {par }}^{2}(\Gamma, M)$. In particular, for $M=\mathbb{C}$ with the trivial action of $\Gamma$, $H_{\text {par }}^{1}(\Gamma, \mathbb{C})$ (and $Z_{\text {par }}^{1}(\Gamma, \mathbb{C})$ ) is isomorphic to the group $\operatorname{Hom}_{0}(\Gamma, \mathbb{C})$, defined in Section 3.

Now let $\Gamma$ act on $P_{k-2}$ via $\left.\right|_{2-k}$ and on $C^{1}\left(\Gamma, P_{k-2}\right)$ via the trivial action. Consider the map $\alpha: C^{1}\left(\Gamma, P_{k-2}\right) \rightarrow C^{1}\left(\Gamma, C^{1}\left(\Gamma, P_{k-2}\right)\right)$ defined as follows: For $\psi: \Gamma \rightarrow P_{k-2}$ we let $\alpha(\psi)$ be a map from $\Gamma$ to $C^{1}\left(\Gamma, P_{k-2}\right)$ such that $\alpha(\psi)(\gamma)$, for $\gamma$ in $\Gamma$, is defined by the formula

$$
\alpha(\psi)(\gamma)(\delta)=\left.(d \psi)(\gamma, \delta)\right|_{2-k} \gamma^{-1}
$$

for all $\delta \in \Gamma$. We then set

$$
\begin{aligned}
& Z_{!}^{1}\left(\Gamma, P_{k-2}\right)=\alpha^{-1}\left(H_{p a r}^{1}\left(\Gamma, Z^{1}\left(\Gamma, P_{k-2}\right)\right)\right), \\
& B_{!}^{1}\left(\Gamma, P_{k-2}\right)=\alpha^{-1}\left(H_{p a r}^{1}\left(\Gamma, B^{1}\left(\Gamma, P_{k-2}\right)\right)\right) .
\end{aligned}
$$

Explicitly, a map $f: \Gamma \rightarrow P_{k-2}$ belongs to $Z_{!}^{1}\left(\Gamma, P_{k-2}\right)$ if and only if

(i) for each $\gamma \in \Gamma,\left.(d f)(\gamma, \delta)\right|_{2-k} \gamma^{-1}$ is a 1-cocycle as a function of $\delta \in \Gamma$,

(ii) $\left.(d f)\left(\gamma_{2} \gamma_{1}, \delta\right)\right|_{2-k}\left(\gamma_{2} \gamma_{1}\right)^{-1}=\left.(d f)\left(\gamma_{2}, \delta\right)\right|_{2-k} \gamma_{2}^{-1}+\left.(d f)\left(\gamma_{1}, \delta\right)\right|_{2-k} \gamma_{1}^{-1}$ for all $\gamma_{1}, \gamma_{2}, \delta$ in $\Gamma$ and

(iii) $(d f)(\pi, \delta) \equiv 0$ for parabolic $\pi$ and all $\delta$ in $\Gamma$.

In other words, $f \in Z_{!}^{1}\left(\Gamma, P_{k-2}\right)$ if and only if

$$
\begin{aligned}
f\left(\gamma_{3} \gamma_{2} \gamma_{1}\right)= & \left.f\left(\gamma_{3} \gamma_{2}\right)\right|_{2-k} \gamma_{1}+f\left(\gamma_{2} \gamma_{1}\right)+\left.f\left(\gamma_{3} \gamma_{1}\right)\right|_{2-k}\left(\gamma_{1}^{-1} \gamma_{2} \gamma_{1}\right) \\
& -\left.f\left(\gamma_{3}\right)\right|_{2-k}\left(\gamma_{2} \gamma_{1}\right)-\left.f\left(\gamma_{2}\right)\right|_{2-k} \gamma_{1}-\left.f\left(\gamma_{1}\right)\right|_{2-k}\left(\gamma_{1}^{-1} \gamma_{2} \gamma_{1}\right)
\end{aligned}
$$

and

$$
f(\delta \pi)=\left.f(\delta)\right|_{2-k} \pi+f(\pi)
$$


Exactly the same is true for $f \in B_{!}^{1}\left(\Gamma, P_{k-2}\right)$ except that, in addition, $\left.(d f)(\gamma, \delta)\right|_{2-k} \gamma^{-1}$ is a 1-coboundary. Therefore $B_{!}^{1}\left(\Gamma, P_{k-2}\right)$ can be canonically embedded in $Z_{!}^{1}\left(\Gamma, P_{k-2}\right)$ and we may define

$$
H_{!}^{1}\left(\Gamma, P_{k-2}\right)=\frac{Z_{!}^{1}\left(\Gamma, P_{k-2}\right)}{B_{!}^{1}\left(\Gamma, P_{k-2}\right)} .
$$

We also set $\bar{S}_{k}^{2}(\Gamma)$ (resp. $\left.\bar{S}_{k}(\Gamma)\right)$ for the space of functions whose complex conjugate is in $S_{k}^{2}(\Gamma)\left(\operatorname{resp} . S_{k}(\Gamma)\right)$ and we associate a map $\tilde{\phi}$ to $F \in \bar{S}_{k}^{2}(\Gamma)$ by setting

$$
\tilde{\phi}(\gamma)=\int_{i}^{\gamma^{-1} i} F(z)(\bar{z}-X)^{k-2} d \bar{z} \quad \text { for all } \gamma \in \Gamma .
$$

With this notation we have

Theorem 7.1. (i) For $k>2$ the maps defined by $\phi$ and $\tilde{\phi}$ induce an isomorphism

$$
\frac{M_{k}^{2}(\Gamma)}{M_{k}(\Gamma)} \oplus \frac{\bar{S}_{k}^{2}(\Gamma)}{\bar{S}_{k}(\Gamma)} \cong H_{!}^{1}\left(\Gamma, P_{k-2}\right) .
$$

(ii) There is an isomorphism

$$
\frac{M_{2}^{2}(\Gamma)}{M_{2}(\Gamma)} \oplus \frac{\bar{S}_{2}^{2}(\Gamma)}{\bar{S}_{2}(\Gamma)} \oplus \mathbb{C} \cong H_{!}^{1}(\Gamma, \mathbb{C})
$$

Proof. (i) We will be using the following formulation of Eichler-Shimura's isomorphism (see [DI, Section 12.2] for a similar formulation):

For each $\phi \in Z^{1}\left(\Gamma, P_{k-2}\right)$ there is a unique pair $\left(g_{1}, \bar{g}_{2}\right) \in M_{k} \times \bar{S}_{k}$ such that

$\phi(\gamma)=\int_{i}^{\gamma^{-1} i} g_{1}(w)(w-X)^{k-2} d w+\int_{i}^{\gamma^{-1} i} \bar{g}_{2}(w)(\bar{w}-X)^{k-2} d \bar{w} \quad$ for all $\gamma \in \Gamma$.

Moreover, if $f$ is the map sending $\phi$ to $\left(g_{1}, \bar{g}_{2}\right)$, then the sequence

$$
0 \rightarrow B^{1}\left(\Gamma, P_{k-2}\right) \stackrel{i}{\hookrightarrow} Z^{1}\left(\Gamma, P_{k-2}\right) \stackrel{f}{\rightarrow} M_{k} \oplus \bar{S}_{k} \rightarrow 0
$$

is exact.

The exact sequence (7.1) can be thought of as a sequence of $\Gamma$-modules with $\Gamma$ acting trivially on each of the modules. This induces an exact sequence of cochain complexes

$$
0 \rightarrow C_{p a r}^{*}\left(\Gamma, B^{1}\left(\Gamma, P_{k-2}\right)\right) \hookrightarrow C_{p a r}^{*}\left(\Gamma, Z^{1}\left(\Gamma, P_{k-2}\right)\right) \rightarrow C_{p a r}^{*}\left(\Gamma, M_{k} \oplus \bar{S}_{k}\right) \rightarrow 0
$$

and this, in turn, induces a long exact sequence

$$
\begin{aligned}
& H_{\text {par }}^{1}\left(\Gamma, B^{1}\left(\Gamma, P_{k-2}\right)\right) \stackrel{i^{*}}{\rightarrow} H_{\text {par }}^{1}\left(\Gamma, Z^{1}\left(\Gamma, P_{k-2}\right)\right) \stackrel{f^{*}}{\rightarrow} H_{\text {par }}^{1}\left(\Gamma, M_{k} \oplus \bar{S}_{k}\right) \\
& \rightarrow H_{p a r}^{2}\left(\Gamma, B^{1}\left(\Gamma, P_{k-2}\right)\right) \rightarrow H_{\text {par }}^{2}\left(\Gamma, Z^{1}\left(\Gamma, P_{k-2}\right)\right) \rightarrow H_{p a r}^{2}\left(\Gamma, M_{k} \oplus \bar{S}_{k}\right) \\
& \rightarrow H^{3}\left(\Gamma, B^{1}\left(\Gamma, P_{k-2}\right)\right) \rightarrow \ldots,
\end{aligned}
$$

where $f^{*}(\psi)(\gamma):=f(\psi(\gamma))$ and $i^{*}$ is induced by the injection $i$ in a similar manner.

Lemma 7.2. $H^{j}(\Gamma, M)=0$ for every $j \geqslant 2$ and every $\mathbb{C}$-vector space $M$. 
Proof. By Selberg's Lemma (cf. Ra or $\mathrm{Se}$ ), there exists a torsion-free subgroup $G$ of finite index in $\Gamma$. Since $G$ is itself a Fuchsian group of the first kind, it can be described through the classical generators and relations. Being torsion-free implies that the only relation is

$$
\left[\gamma_{1}, \gamma_{g+1}\right] \ldots\left[\gamma_{g}, \gamma_{2 g}\right] \pi_{1} \ldots \pi_{p}=1
$$

where $\gamma_{j}$ (resp. $\pi_{l}$ ) are hyperbolic (resp. parabolic) generators of $G$. Therefore, $G$ is freely generated by $\gamma_{1}, \ldots, \gamma_{2 g}$ and $\pi_{1}, \ldots, \pi_{p-1}$. Since the cohomological dimension of a free group is 1 , an application of the transfer operator ( $\mathrm{B}$, Proposition 10.1 of III) implies that $H^{j}(\Gamma, M)=0$ for all $j \geqslant 2$. This completes the proof of the lemma.

We also have (see $\underline{\operatorname{Sh}}$, Chapter 8)

$$
H_{\text {par }}^{2}(\Gamma, M) \cong M / M_{1}
$$

where $M_{1}$ is the subspace of $M$ generated by $M .(\gamma-1)$ for all $\gamma$ in $\Gamma$. Therefore (7.2) implies the exact sequence

$$
\begin{aligned}
0 & \rightarrow i^{*}\left(H_{p a r}^{1}\left(\Gamma, B^{1}\left(\Gamma, P_{k-2}\right)\right)\right) \hookrightarrow H_{p a r}^{1}\left(\Gamma, Z^{1}\left(\Gamma, P_{k-2}\right)\right) \stackrel{f^{*}}{\rightarrow} H_{p a r}^{1}\left(\Gamma, M_{k} \oplus \bar{S}_{k}\right) \\
& \rightarrow B^{1}\left(\Gamma, P_{k-2}\right) \rightarrow Z^{1}\left(\Gamma, P_{k-2}\right) \rightarrow M_{k} \oplus \bar{S}_{k} \rightarrow 0 .
\end{aligned}
$$

In particular, this implies that

$\operatorname{dim}\left(i^{*}\left(H_{\text {par }}^{1}\left(\Gamma, B^{1}\left(\Gamma, P_{k-2}\right)\right)\right)\right)-\operatorname{dim}\left(H_{\text {par }}^{1}\left(\Gamma, Z^{1}\left(\Gamma, P_{k-2}\right)\right)\right)+\cdots-\operatorname{dim}\left(M_{k} \oplus \bar{S}_{k}\right)=0$.

Because of (7.1), the last three dimensions cancel out and we are left with

$$
\begin{aligned}
\operatorname{dim}\left(H_{\text {par }}^{1}\left(\Gamma, Z^{1}\left(\Gamma, P_{k-2}\right)\right)\right)-\operatorname{dim}\left(i^{*}\left(H_{\text {par }}^{1}\left(\Gamma, B^{1}\left(\Gamma, P_{k-2}\right)\right)\right)\right) & \\
& =\operatorname{dim}\left(H_{\text {par }}^{1}\left(\Gamma, M_{k} \oplus \bar{S}_{k}\right)\right) .
\end{aligned}
$$

From this we conclude that $f^{*}$ induces an isomorphism

$$
\frac{H_{p a r}^{1}\left(\Gamma, Z^{1}\left(\Gamma, P_{k-2}\right)\right)}{i^{*}\left(H_{p a r}^{1}\left(\Gamma, B^{1}\left(\Gamma, P_{k-2}\right)\right)\right)} \cong H_{p a r}^{1}\left(\Gamma, M_{k} \oplus \bar{S}_{k}\right) .
$$

Because of the relations (2.1) and the vanishing of homomorphisms at elements of finite order, each element of $H_{p a r}^{1}\left(\Gamma, M_{k} \oplus \bar{S}_{k}\right)$ is uniquely determined by its values at the $2 g$ hyperbolic generators of $\Gamma$. Therefore,

$$
H_{\text {par }}^{1}\left(\Gamma, M_{k} \oplus \bar{S}_{k}\right) \cong \bigoplus_{i=1}^{2 g}\left(M_{k} \oplus \bar{S}_{k}\right) .
$$

On the other hand, as it was pointed out in [CDO, we have exact sequences

$$
\begin{gathered}
0 \hookrightarrow S_{k} \rightarrow S_{k}^{2} \stackrel{\psi}{\rightarrow} \bigoplus_{i=1}^{2 g} S_{k}, \\
0 \hookrightarrow M_{k} \rightarrow M_{k}^{2} \stackrel{\psi}{\rightarrow} \bigoplus_{i=1}^{2 g} M_{k},
\end{gathered}
$$

where $\psi$ sends a form $F$ to the vector $\left(\left.F\right|_{k}\left(\gamma_{1}^{-1}-1\right), \ldots,\left.F\right|_{k}\left(\gamma_{2 g}^{-1}-1\right)\right)$, (the $\gamma_{i}$ 's being the hyperbolic generators of $\Gamma$ that we fixed in Section 2). Theorems 2.1 and 2.2 allow us now to show, by comparison of dimensions, that, in addition, $\psi$ is onto in both exact sequences for $k>2$. Therefore, the right-hand side of (7.3) 
is isomorphic to $M_{k}^{2} / M_{k} \oplus \bar{S}_{k}^{2} / \bar{S}_{k}$ via the map $\psi \times \tilde{\psi}$, where $\tilde{\psi}(f)$ is defined by $\tilde{\psi}(f):=\overline{\psi(\bar{f})}$ (the complex conjugation on the right-hand side being understood component-wise). Therefore,

$$
\frac{H_{\text {par }}^{1}\left(\Gamma, Z^{1}\left(\Gamma, P_{k-2}\right)\right)}{i^{*}\left(H_{\text {par }}^{1}\left(\Gamma, B^{1}\left(\Gamma, P_{k-2}\right)\right)\right)} \cong H_{\text {par }}^{1}\left(\Gamma, M_{k} \oplus \bar{S}_{k}\right) \cong \frac{M_{k}^{2}}{M_{k}} \oplus \frac{\bar{S}_{k}^{2}}{\bar{S}_{k}}
$$

Finally,

Lemma 7.3. The sequence

$$
0 \rightarrow Z^{1}\left(\Gamma, P_{k-2}\right) \hookrightarrow Z_{!}^{1}\left(\Gamma, P_{k-2}\right) \stackrel{\alpha}{\rightarrow} H_{p a r}^{1}\left(\Gamma, Z^{1}\left(\Gamma, P_{k-2}\right)\right) \rightarrow 0
$$

is exact.

Proof. Since $\alpha(\psi) \equiv 0$ if and only if $d \psi \equiv 0, \operatorname{ker}(\alpha)=Z^{1}\left(\Gamma, P_{k-2}\right)$. On the other hand, by the definition of $Z_{!}^{1}\left(\Gamma, P_{k-2}\right)$, we have $\operatorname{im}(\alpha) \subset H_{p a r}^{1}\left(\Gamma, Z^{1}\left(\Gamma, P_{k-2}\right)\right)$. If $\chi \in H_{\text {par }}^{1}\left(\Gamma, Z^{1}\left(\Gamma, P_{k-2}\right)\right)$, an easy computation implies that $\left.\chi(\gamma)(\delta)\right|_{2-k} \gamma$ gives rise to a 2-cocycle. From Lemma 7.2, $H^{2}\left(\Gamma, P_{k-2}\right)=0$ and thus, there exists a $\phi: \Gamma \rightarrow P_{k-2}$ such that $\left.(d \phi)(\gamma, \delta)\right|_{2-k} \gamma^{-1}=\chi(\gamma)(\delta)$; i.e. $\chi=\alpha(\phi)$ and $\phi \in Z_{1}^{1}$. This shows that $H_{\text {par }}^{1}\left(\Gamma, Z^{1}\left(\Gamma, P_{k-2}\right)\right) \subset \operatorname{im}(\alpha)$.

In the same way we can show the exactness of the sequence:

$$
0 \rightarrow Z^{1}\left(\Gamma, P_{k-2}\right) \hookrightarrow B_{!}^{1}\left(\Gamma, P_{k-2}\right) \stackrel{\alpha}{\rightarrow} H_{p a r}^{1}\left(\Gamma, B^{1}\left(\Gamma, P_{k-2}\right)\right) \rightarrow 0 .
$$

The last two sequences in combination with (7.5) imply the isomorphism

$$
\frac{M_{k}^{2}}{M_{k}} \oplus \frac{\bar{S}_{k}^{2}}{\bar{S}_{k}} \cong\left(\frac{Z_{!}^{1}\left(\Gamma, P_{k-2}\right)}{Z^{1}\left(\Gamma, P_{k-2}\right)}\right) /\left(\frac{B_{!}^{1}\left(\Gamma, P_{k-2}\right)}{Z^{1}\left(\Gamma, P_{k-2}\right)}\right) \cong \frac{Z_{!}^{1}\left(\Gamma, P_{k-2}\right)}{B_{!}^{1}\left(\Gamma, P_{k-2}\right)} .
$$

To show that this isomorphism is induced by $\phi \times \tilde{\phi}$ we unravel the definitions of our maps: $F \in M_{k}^{2}$ is first mapped, via $\psi$ to $\left(\left.F\right|_{k}\left(\gamma_{i}-1\right)\right)_{i=1}^{2 g}$, or, equivalently, to $\left.\gamma \mapsto F\right|_{k}\left(\gamma^{-1}-1\right)$. This is mapped to an $F_{1} \in \operatorname{Hom}\left(\Gamma, Z^{1}\left(\Gamma, P_{k-2}\right)\right)$ such that $f^{*}\left(F_{1}\right)(\gamma)=\left.F\right|_{k}\left(\gamma^{-1}-1\right)$. By the definition of $f^{*}$ we have $f\left(F_{1}(\gamma)\right)=\left.F\right|_{k}\left(\gamma^{-1}-1\right)$ and hence

$$
F_{1}(\gamma)(\delta)=\int_{i}^{\delta^{-1} i}\left(\left.F\right|_{k}\left(\gamma^{-1}-1\right)\right)(w)(w-X)^{k-2} d w
$$

To determine the image of $F_{1}$ in $H_{!}^{1}$ we identify an element $\psi \in Z_{!}^{1}$ such that $\left.(d \psi)(\gamma, \delta)\right|_{2-k} \gamma^{-1}=F_{1}(\gamma)(\delta)$. We verify that the $\phi$ given in the beginning of the section is such a map as follows. The change of variables $w \rightarrow \gamma^{-1} w$ in the integral

$$
\phi(\delta \gamma)-\phi(\gamma)=\int_{\gamma^{-1} i}^{\gamma^{-1} \delta^{-1} i} F(w)(w-X)^{k-2} d w
$$

gives

$$
\begin{aligned}
\int_{i}^{\delta^{-1} i} F\left(\gamma^{-1} w\right)\left(\gamma^{-1} w-\right. & X)^{k-2} d\left(\gamma^{-1} w\right) \\
& =\int_{i}^{\delta^{-1} i}\left(\left.F\right|_{k} \gamma^{-1}\right)(w) j\left(\gamma^{-1}, w\right)^{k-2}\left(\gamma^{-1} w-X\right)^{k-2} d w
\end{aligned}
$$

where we used $d(\gamma w)=\frac{d w}{j(\gamma, w)^{2}}$. This, in combination with the identity

$$
(w-\gamma X) j(\gamma, X)=\left(\gamma^{-1} w-X\right) j\left(\gamma^{-1}, w\right),
$$


implies

$$
(d \phi)(\gamma, \delta)=\phi(\delta \gamma)-\left.\phi(\delta)\right|_{2-k} \gamma-\phi(\gamma)=\left.\left[\left.\int_{i}^{\delta^{-1} i} F\right|_{k}\left(\gamma^{-1}-1\right)(w)(w-X)^{k-2} d w\right]\right|_{2-k} \gamma
$$

We work in a similar way for $\tilde{\phi}$ and $\bar{S}_{k}^{2}$.

(ii) We work in the same way for $k=2$. The only difference is at (7.5). Specifically, because of Theorems 2.1 and 2.2 , the exact sequences (7.4) imply

$$
\frac{M_{2}^{2}}{M_{2}} \oplus\left(\frac{\bar{S}_{2}^{2}}{\bar{S}_{2}} \oplus \mathbb{C}\right) \cong \bigoplus_{i=1}^{2 g}\left(M_{2} \oplus \bar{S}_{2}\right) .
$$

Therefore,

$$
\frac{H_{\text {par }}^{1}\left(\Gamma, Z^{1}(\Gamma, \mathbb{C})\right)}{i^{*}\left(H_{\text {par }}^{1}\left(\Gamma, B^{1}(\Gamma, \mathbb{C})\right)\right)} \cong H_{\text {par }}^{1}\left(\Gamma, M_{2} \oplus \bar{S}_{2}\right) \cong \frac{M_{2}^{2}}{M_{2}} \oplus \frac{\bar{S}_{2}^{2}}{\bar{S}_{2}} \oplus \mathbb{C} .
$$

As in the proof of part (i), this implies the desired isomorphism and completes the proof of Theorem 7.1.

\section{Spectral theory}

Before proceeding to the proofs of Propositions A, B, C and D, in Sections 9,10 and 11, we gather here results we shall require from the spectral theory of automorphic forms.

We will study the series $U_{\mathfrak{a} m}(z, s, k)$ and $Q_{\mathfrak{a} m}(z, s, 1 ; \bar{f})$ by means of their spectral expansions, which we now recall (see, for example, [I1] and the references therein for further background information and complete proofs). The hyperbolic Laplacian $\Delta=-4 y^{2} d / d z d / d \bar{z}$ operates on $L^{2}(\Gamma \backslash \mathfrak{H})$, the space of smooth, automorphic, square integrable functions. Any element $\xi$ of $L^{2}(\Gamma \backslash \mathfrak{H})$ may be decomposed into constituent parts from the discrete and continuous spectrum of $\Delta$. This RoelckeSelberg decomposition amounts to the identity

$$
\xi(z)=\sum_{j=0}^{\infty}\left\langle\xi, \eta_{j}\right\rangle \eta_{j}(z)+\frac{1}{4 \pi} \sum_{\mathfrak{b}} \int_{-\infty}^{\infty}\left\langle\xi, E_{\mathfrak{b}}(\cdot, 1 / 2+i r)\right\rangle E_{\mathfrak{b}}(z, 1 / 2+i r) d r
$$

where $\left\{\eta_{j}\right\}$ denotes a complete orthonormal basis of Maass forms, with corresponding eigenvalues $\lambda_{j}=s_{j}\left(1-s_{j}\right)$, which forms the discrete spectrum. For notational convenience, we write $\langle\cdot, \cdot\rangle$ here for the inner product on $\Gamma \backslash \mathfrak{H}$ of weight zero forms (i.e., $\Gamma$-invariant functions). As always, we will write $s_{j}=\sigma_{j}+i t_{j}$, chosen so that $\sigma_{j} \geqslant 1 / 2$ and $t_{j} \geqslant 0$, and we enumerate the eigenvalues, counted with multiplicity, by $0=\lambda_{0}<\lambda_{1} \leqslant \lambda_{2} \leqslant \cdots$. Recall that Weyl's law ((11.3) of [11]) implies

$$
\#\left\{j|| \lambda_{j} \mid \leqslant T\right\} \ll T .
$$

The decomposition (8.1) is absolutely convergent for each fixed $z$ and uniform on compact subsets of $\mathfrak{H}$, provided $\xi$ and $\Delta \xi$ are smooth and bounded (see, for example, Theorem 4.7 and Theorem 7.3 of [I1]).

For each $j$, the Fourier expansion of $\eta_{j}$ is

$$
\eta_{j}\left(\sigma_{\mathfrak{a}} z\right)=\rho_{\mathfrak{a} j}(0) y^{1-s_{j}}+\sum_{m \neq 0} \rho_{\mathfrak{a} j}(m) W_{s_{j}}(m z) .
$$


For all but finitely many of the $j$ (corresponding to $\lambda_{j}<1 / 4$ ) we have $\sigma_{j}=1 / 2$ and $\rho_{\mathfrak{a} j}(0)=0$. The constant $\delta_{\Gamma}$ used in Propositions B, C and D, and throughout this paper is chosen so that $1-\delta_{\Gamma}>\sigma_{1} \geqslant 1 / 2$.

We have the bounds

$$
\begin{aligned}
\rho_{\mathfrak{a} j}(0) & \ll 1, \\
\rho_{\mathfrak{a} j}(m) & \ll \frac{\left|t_{j}\right|}{\sqrt{|m|}} e^{\pi\left|t_{j}\right| / 2}, \quad m \neq 0,
\end{aligned}
$$

where the implied constant depends only on $\Gamma$. The estimate (8.4) is true because, again, there are only finitely many $j$ with $\rho_{\mathfrak{a} j}(0) \neq 0$. The estimate (8.5) follows from the formula of Bruggeman and Kuznetsov, as stated in (9.13) of [1]. See (8.6) of [JO for the simple derivation of (8.5). We also have, for any $k \geqslant 0$ and $\sigma=\operatorname{Re}(s)>1 / 2-k$, the bound

$$
W_{s}(n z) \ll \frac{|s|^{2 k}+1}{(|n| y)^{2 k-1+\sigma}}|\Gamma(s)|
$$

from [JO, (8.11)] (with implied constant depending solely on $\sigma$ and $k$ ) and Stirling's classical formula

$$
|\Gamma(\sigma+i t)| \sim \sqrt{2 \pi}|t|^{\sigma-1 / 2} e^{-\pi|t| / 2} \quad \text { as }|t| \rightarrow \infty .
$$

Next we bound $\eta_{j}(z)$. Combine (8.4), (8.5), (8.6), (8.7) and the Fourier expansion (8.3) to obtain

$$
\eta_{j}\left(\sigma_{\mathfrak{a}} z\right) \ll y^{1 / 2}+\left(\left|t_{j}\right|^{7 / 2}+1\right) y^{-3 / 2} \sum_{m \neq 0}|m|^{-2}
$$

as $y \mapsto \infty$. Therefore

$$
\eta_{j}(z) \ll y_{\Gamma}(z)^{1 / 2}+\left(\left|t_{j}\right|^{7 / 2}+1\right) y_{\Gamma}(z)^{-3 / 2}
$$

for an implied constant depending on $\Gamma$ alone. This is (8.13) of [JO].

Recall the Fourier expansion of $E_{\mathfrak{a}}(z, s)$ in (4.5). The analogues of (8.4) and (8.5) for the continuous spectrum are (we will always assume $T \geqslant 0$ for simplicity)

$$
\begin{aligned}
\left|\phi_{\mathfrak{a} \mathfrak{b}}(1 / 2+i r)\right| & \leqslant 1, \\
\int_{T}^{T+1}\left|\phi_{\mathfrak{a} \mathfrak{b}}(m, 1 / 2+i r)\right|^{2} d r & \ll \frac{T^{2}}{|m|} e^{\pi T},
\end{aligned}
$$

where (8.9) follows from (6.28) of [1], and (8.10) again from the Bruggeman and Kuznetsov formula as in (8.7) of [JO]. Another useful direct bound, as shown in [JO, Lemma 8.4], is

$$
\phi_{\mathfrak{a} \mathfrak{b}}(m, 1 / 2+i r) \ll|m|^{2},
$$

for $r$ in $[T, T+1]$ and an implied constant depending on $T$ and $\Gamma$ alone.

Let $C^{\infty}(\Gamma \backslash \mathfrak{H}, k)$ denote the space of smooth functions $\psi$ on $\mathfrak{H}$ that transform as

$$
\psi(\gamma z)=\varepsilon(\gamma, z)^{k} \psi(z)
$$

for $\gamma$ in $\Gamma$ and $\varepsilon(\gamma, z)=j(\gamma, z) /|j(\gamma, z)|$. For example, $U_{\mathfrak{a} m}(z, s, k) \in C^{\infty}(\Gamma \backslash \mathfrak{H}, k)$. It should be clear from the context whether we mean this new notion of weight or the previous definition of weight. Trivially, if $\psi \in C^{\infty}(\Gamma \backslash \mathfrak{H}, k)$, then $|\psi|$ has weight zero (in either definition). We define the Maass raising and lowering operators by

$$
R_{k}=2 i y \frac{d}{d z}+\frac{k}{2}, L_{k}=-2 i y \frac{d}{d \bar{z}}-\frac{k}{2} \text {. }
$$


It is an elementary exercise to show that

$$
R_{k}: C^{\infty}(\Gamma \backslash \mathfrak{H}, k) \rightarrow C^{\infty}(\Gamma \backslash \mathfrak{H}, k+2), L_{k}: C^{\infty}(\Gamma \backslash \mathfrak{H}, k) \rightarrow C^{\infty}(\Gamma \backslash \mathfrak{H}, k-2) .
$$

For $n>0$, we write $R^{n}$ for $R_{k+2 n-2} \cdots R_{k+2} R_{k}$ and $L^{n}$ for $L_{k-2 n+2} \cdots L_{k-2} L_{k}$. (To simplify, we omit $k$ from the notation of the operators $L^{n}$ and $R^{n}$. It will usually be clear in each case.) We also let $L^{0}$ and $R^{0}$ be the identity operator.

The hyperbolic Laplacian $\Delta$ can be realized as

$$
\Delta=-L_{2} R_{0}=-R_{-2} L_{0}
$$

By a direct calculation (see also Lemma 9.2 of [JO]),

$$
\begin{aligned}
R_{k} U_{\mathfrak{a} m}(z, s, k) & =(s+k / 2) U_{\mathfrak{a} m}(z, s, k+2)-4 \pi m U_{\mathfrak{a} m}(z, s+1, k+2), \\
L_{k} U_{\mathfrak{a} m}(z, s, k) & =(s-k / 2) U_{\mathfrak{a} m}(z, s, k-2) .
\end{aligned}
$$

To see what happens to the Fourier expansions when we raise or lower the weight we need the next lemma. Set

$$
\omega(n, m, i, j)=(-4 \pi m)^{i}\left(\frac{m}{|m|}\right)^{j} \frac{(2 n) !}{(i+j) !(i-j) !(n-i) !} .
$$

Lemma 8.1. For $n \geqslant 0$ we have

$$
\begin{aligned}
R^{n}\left(W_{s}(m z)\right) & =\sum_{i=0}^{n} \sum_{j=-i}^{i} \omega(n, m, i, j) y^{i} W_{s+j}(m z), \\
L^{n}\left(W_{s}(m z)\right) & =\sum_{i=0}^{n} \sum_{j=-i}^{i} \omega(n,-m, i, j) y^{i} W_{s+j}(m z), \\
R^{n}\left(y^{s}\right)=L^{n}\left(y^{s}\right) & =s(s+1) \cdots(s+n-1) y^{s} .
\end{aligned}
$$

Proof. See Sections 4 and 5 of [02] for the proofs of (8.15) and (8.16). A simple computation gives (8.17).

It follows from this lemma that

$$
R^{n}\left(W_{s}(m z)\right), L^{n}\left(W_{s}(m z)\right) \ll \sum_{i=0}^{n} \sum_{j=-i}^{i}(|m| y)^{i}\left|W_{s+j}(m z)\right| .
$$

Combine this with (8.6) to prove that for any $s$ with $1 / 2 \leqslant \operatorname{Re}(s) \leqslant 1-\delta, \delta>0$ and any $l \geqslant 0$,

$$
R^{n}\left(W_{s}(m z)\right), L^{n}\left(W_{s}(m z)\right) \ll(|m| y)^{n-2 l-3 / 2}\left(|s|^{2 l+2+n}+1\right)|\Gamma(s)|
$$

as $y \rightarrow \infty$. The implied constant depends on $n, l$ and $\delta$.

Lemma 8.2. For $n \geqslant 0$ we have

$$
R^{n}\left(\eta_{j}(z)\right), L^{n}\left(\eta_{j}(z)\right) \ll\left(\left|t_{j}\right|^{n}+1\right) y_{\Gamma}(z)^{1 / 2}+\left(\left|t_{j}\right|^{2 n+5}+1\right) y_{\Gamma}(z)^{-3 / 2}
$$

with the implied constant depending on $n$ and $\Gamma$ alone.

Proof. First consider

$$
\rho_{\mathfrak{a} j}(0) R^{n}\left(y^{1-s_{j}}\right)+\sum_{m \neq 0} \rho_{\mathfrak{a} j}(m) R^{n}\left(W_{s_{j}}(m z)\right) .
$$

With $(8.4),(8.5),(8.7),(8.17)$ and (8.18) we see that (8.19) is uniformly convergent in $z$ and bounded by a constant times

$$
\left(\left|t_{j}\right|^{n}+1\right) y^{1 / 2}+\left(\left|t_{j}\right|^{2 n+5}+1\right) y^{-3 / 2}
$$


as $y \rightarrow \infty$. Thus (8.19) must equal $R^{n}\left(\eta_{j}\left(\sigma_{\mathfrak{a}} z\right)\right)$. To get from this to the statement of the lemma it is easiest to introduce the following operator. For $\tau \in P S L_{2}(\mathbb{R})$, let the operator $\theta_{\tau, k}: C^{\infty}(\Gamma \backslash \mathfrak{H}, k) \rightarrow C^{\infty}\left(\tau^{-1} \Gamma \tau \backslash \mathfrak{H}, k\right)$ have the action

$$
\theta_{\tau, k} \psi(z)=\frac{\psi(\tau z)}{\varepsilon(\tau, z)^{k}}
$$

We will need the easily verified fact that $\theta$ commutes with the raising and lowering operators:

$$
\begin{aligned}
& \theta_{\tau, k-2} L_{k}=L_{k} \theta_{\tau, k}, \\
& \theta_{\tau, k+2} R_{k}=R_{k} \theta_{\tau, k} .
\end{aligned}
$$

Now we can say that

$$
\left|\left(R^{n} \eta_{j}(w)\right)\right|_{w=\sigma_{\mathfrak{a}} z}|=| \theta_{\sigma_{\mathfrak{a}}, 2 n} R^{n} \eta_{j}(z)|=| R^{n} \theta_{\sigma_{\mathfrak{a}}, 0} \eta_{j}(z)|=| R^{n}\left(\eta_{j}\left(\sigma_{\mathfrak{a}} z\right)\right) \mid .
$$

A similar result holds for the lowering operator $L^{n}$ and the lemma follows.

Lemma 8.3. For $n \geqslant 0$ we have

$$
\int_{T}^{T+1}\left|R^{n} E_{\mathfrak{a}}(z, 1 / 2+i r)\right|^{2} d r, \quad \int_{T}^{T+1}\left|L^{n} E_{\mathfrak{a}}(z, 1 / 2+i r)\right|^{2} d r \ll T^{4 n+12} y_{\Gamma}(z) .
$$

Proof. With (4.6) and (8.18) we see that, for $r$ in $[T, T+1]$ and any integer $l \geqslant 0$,

$$
\begin{aligned}
R^{n} E_{\mathfrak{a}}\left(\sigma_{\mathfrak{b}} z, 1 / 2+i r\right) \ll & T^{n} y^{1 / 2} \\
& +\sum_{m \neq 0}\left|\phi_{\mathfrak{a} \mathfrak{b}}(m, 1 / 2+i r)\right|(|m| y)^{n-2 l-3 / 2} T^{2 l+2+n} e^{-\pi T / 2},
\end{aligned}
$$

so that

$$
\begin{aligned}
& \int_{T}^{T+1}\left|R^{n} E_{\mathfrak{a}}\left(\sigma_{\mathfrak{b}} z, 1 / 2+i r\right)\right|^{2} d r \ll T^{2 n} y \\
+ & T^{2 n+2 l+2} e^{-\pi T / 2} y^{n-2 l-1} \int_{T}^{T+1} \sum_{m \neq 0}\left|\phi_{\mathfrak{a} \mathfrak{b}}(m, 1 / 2+i r)\right||m|^{n-2 l-3 / 2} d r \\
& +T^{2 n+4 l+4} e^{-\pi T} y^{2 n-4 l-3} \\
& \times \int_{T}^{T+1} \sum_{m_{1}, m_{2} \neq 0}\left|\phi_{\mathfrak{a} \mathfrak{b}}\left(m_{1}, 1 / 2+i r\right) \phi_{\mathfrak{a} \mathfrak{b}}\left(m_{2}, 1 / 2+i r\right)\right|\left|m_{1} m_{2}\right|^{n-2 l-3 / 2} d r .
\end{aligned}
$$

With (8.11) it is clear that the sums in the integrals on the right side of (8.23) are absolutely convergent for $l=\lceil n / 2+1\rceil$ and uniformly bounded for $T \leqslant r \leqslant T+1$. Interchanging sums and integrals in (8.23) is now justified with Corollary 8.6 below, and with (8.10) and the Cauchy-Schwarz inequality we obtain the lemma for the raising operator. The argument for $L^{n}$ is identical.

We shall have frequent need of the next three standard analysis results.

Theorem 8.4. Let $z$ be in a set $S \subseteq \mathbb{C}$. Suppose the functions $f_{k}(z)$ are smooth and $\sum_{k=1}^{\infty} f_{k}(z)$ converges pointwise on $S$. If $\sum_{k=1}^{\infty} \frac{d}{d z} f_{k}(z)$ converges uniformly, then

$$
\frac{d}{d z} \sum_{k=1}^{\infty} f_{k}(z)=\sum_{k=1}^{\infty} \frac{d}{d z} f_{k}(z)
$$


Theorem 8.5. Suppose the functions $g_{k}(r)$ are integrable on $[a, b]$ and $\lim _{k \rightarrow \infty} g_{k}(r)$ converges pointwise. Then

$$
\lim _{k \rightarrow \infty} \int_{a}^{b} g_{k}(r) d r=\int_{a}^{b} \lim _{k \rightarrow \infty} g_{k}(r) d r
$$

if, for all $k,\left|g_{k}(r)\right| \leqslant C$ for some fixed constant $C$.

Corollary 8.6. Suppose the functions $h_{k}(r)$ are smooth on $[a, b]$ and $\sum_{k=1}^{\infty}\left|h_{k}(r)\right|$ exists and is uniformly bounded on $[a, b]$. Then

$$
\sum_{k=1}^{\infty} \int_{a}^{b} h_{k}(r) d r=\int_{a}^{b}\left(\sum_{k=1}^{\infty} h_{k}(r)\right) d r .
$$

Theorem 8.4 is a weak form of Theorem 7.17 in $[\mathrm{Ru}$. Theorem 8.5 follows from Lebesgue's Dominated Convergence Theorem as in [Ru, Theorem 10.32]. The corollary follows directly from Theorem 8.5.

\section{Proof of Proposition A}

Proposition A. For $k \in 2 \mathbb{Z}$ and $\sigma=\operatorname{Re}(s)>1$, the series $U_{\mathfrak{a} m}(z, s, k)$, $Q_{\mathfrak{a} m}(z, s, 1 ; \bar{f}), Q_{\mathfrak{a} m}^{\prime}(z, s, 1 ; \bar{f})$ and $G_{\mathfrak{a} m}(z, s-1 ; \bar{F})$ converge absolutely and uniformly on compact sets to analytic functions of $s$. For these $s$ we have

$$
\begin{aligned}
U_{\mathfrak{a} 0}(z, s, k) & \ll y_{\Gamma}(z)^{\sigma}, \\
U_{\mathfrak{a} m}(z, s, k) & \ll 1, \quad m>0, \\
Q_{\mathfrak{a} m}(z, s, 1 ; \bar{f}) & \ll y_{\Gamma}(z)^{1 / 2-\sigma / 2}, \quad m \geqslant 0, \\
y Q_{\mathfrak{a} m}^{\prime}(z, s, 1 ; \bar{f}) & \ll(|m|+1) y_{\Gamma}(z)^{1 / 2-\sigma / 2}, \quad m \geqslant 0, \\
y G_{\mathfrak{a} m}(z, s-1 ; \bar{F}) & \ll y_{\Gamma}(z)^{1 / 2-\sigma / 2}, \quad m \geqslant 0,
\end{aligned}
$$

where the implied constants depend on $s, k, f$ and $\Gamma$ but not on $m$.

Proof. The Eisenstein series $E_{\mathfrak{a}}(z, s)$ given by (4.5) equals $U_{\mathfrak{a} 0}(z, s, 0)$, is absolutely convergent for $\operatorname{Re}(s)>1$ and satisfies

$$
E_{\mathfrak{a}}\left(\sigma_{\mathfrak{b}} z, s\right)=\delta_{\mathfrak{a} \mathfrak{b}} y^{s}+\phi_{\mathfrak{a} \mathfrak{b}}(s) y^{1-s}+O\left(e^{-2 \pi y}\right)
$$

as $y \rightarrow \infty$ by (4.7). Hence

$$
U_{\mathfrak{a} 0}(z, s, k) \ll E_{\mathfrak{a}}(z, \sigma) \ll y_{\Gamma}(z)^{\sigma},
$$

which is (i).

For $m>0$ we have (with $\left|e\left(m \sigma_{\mathfrak{a}}{ }^{-1} \gamma z\right)\right| \leqslant 1$ )

$$
\begin{aligned}
U_{\mathfrak{a} m}\left(\sigma_{\mathfrak{a}} z, s, k\right) & \ll y^{\sigma} e^{-2 \pi m y}+\sum_{\substack{\gamma \in \Gamma_{\mathfrak{a}} \backslash \Gamma \\
\gamma \neq i \text { dentity }}} \operatorname{Im}\left(\sigma_{\mathfrak{a}}{ }^{-1} \gamma \sigma_{\mathfrak{a}} z\right)^{\sigma} \\
& \ll y^{\sigma} e^{-2 \pi m y}+\left|E_{\mathfrak{a}}\left(\sigma_{\mathfrak{a}} z, \sigma\right)-y^{\sigma}\right| \ll 1 .
\end{aligned}
$$

At any other cusp $\mathfrak{b} \neq \mathfrak{a}$,

$$
U_{\mathfrak{a} m}\left(\sigma_{\mathfrak{b}} z, s, k\right) \ll E_{\mathfrak{a}}\left(\sigma_{\mathfrak{b}} z, \sigma\right) \ll \phi_{\mathfrak{a} \mathfrak{b}}(s) y^{1-\sigma} \ll 1 .
$$

We have shown statement (ii).

By Lemma 4.1 and the subsequent discussion we have, for any $\epsilon>0$,

$$
F_{\mathfrak{a}}\left(\sigma_{\mathfrak{b}} z\right) \ll y^{\epsilon}+y^{-\epsilon}+1
$$


for all $z$ in $\mathfrak{H}$. It is then apparent that

$$
F_{\mathfrak{a}}\left(\gamma \sigma_{\mathfrak{b}} z\right)=F_{\mathfrak{a}}\left(\sigma_{\mathfrak{a}} \sigma_{\mathfrak{a}}{ }^{-1} \gamma \sigma_{\mathfrak{b}} z\right) \ll \operatorname{Im}\left(\sigma_{\mathfrak{a}}{ }^{-1} \gamma \sigma_{\mathfrak{b}} z\right)^{\epsilon}+\operatorname{Im}\left({\sigma_{\mathfrak{a}}}^{-1} \gamma \sigma_{\mathfrak{b}} z\right)^{-\epsilon}+1
$$

for any cusp $\mathfrak{b}$ and any $z$ in $\mathfrak{H}$. The implied constant depends solely on $\epsilon, f$ and $\Gamma$. In the case $\mathfrak{a}=\mathfrak{b}$ we may improve (9.2). The Fourier expansion of $F_{\mathfrak{a}}$ yields

$$
F_{\mathfrak{a}}\left(\sigma_{\mathfrak{b}} z\right)=\int_{\mathfrak{a}}^{\mathfrak{b}} f(w) d w+\frac{1}{2 \pi i} \sum_{n=1}^{\infty} \frac{a_{\mathfrak{b}}(n)}{n} e(n z)
$$

with $a_{\mathfrak{b}}(n)$ the $n$th Fourier coefficient of $f$ at the cusp $\mathfrak{b}$. Thus, when $\mathfrak{a}=\mathfrak{b}$,

$$
F_{\mathfrak{a}}\left(\sigma_{\mathfrak{a}} z\right) \ll e^{-2 \pi y} \text { as } y \rightarrow \infty .
$$

Consequently,

$$
\begin{aligned}
Q_{\mathfrak{a} m}\left(\sigma_{\mathfrak{a}} z, s, 1 ; \bar{f}\right) \ll & \sum_{\gamma \in \Gamma_{\mathfrak{a}} \backslash \Gamma}\left|F_{\mathfrak{a}}\left(\gamma \sigma_{\mathfrak{a}} z\right)\right| \operatorname{Im}\left(\sigma_{\mathfrak{a}}{ }^{-1} \gamma \sigma_{\mathfrak{a}} z\right)^{\sigma} \\
\ll & e^{-2 \pi y} y^{\sigma}+\sum_{\substack{\gamma \in \Gamma_{\mathfrak{a}} \backslash \Gamma \\
\gamma \neq \text { identity }}}\left(\operatorname{Im}\left(\sigma_{\mathfrak{a}}{ }^{-1} \gamma \sigma_{\mathfrak{a}} z\right)^{\sigma+\epsilon}\right. \\
& \left.+\operatorname{Im}\left(\sigma_{\mathfrak{a}}{ }^{-1} \gamma \sigma_{\mathfrak{a}} z\right)^{\sigma-\epsilon}+\operatorname{Im}\left(\sigma_{\mathfrak{a}}^{-1} \gamma \sigma_{\mathfrak{a}} z\right)^{\sigma}\right) \\
\ll & y^{1-\sigma+\epsilon}
\end{aligned}
$$

for $\sigma>1+\epsilon$ as $y \rightarrow \infty$ by (9.1). When $\mathfrak{a} \neq \mathfrak{b}$ we do not need to worry about the $y^{s}$ term and

$$
\begin{aligned}
Q_{\mathfrak{a} m}\left(\sigma_{\mathfrak{b}} z, s, 1 ; \bar{f}\right) & \ll E_{\mathfrak{a}}\left(\sigma_{\mathfrak{b}} z, \sigma+\epsilon\right)+E_{\mathfrak{a}}\left(\sigma_{\mathfrak{b}} z, \sigma-\epsilon\right)+E_{\mathfrak{a}}\left(\sigma_{\mathfrak{b}} z, \sigma\right) \\
& \ll y^{1-\sigma+\epsilon}
\end{aligned}
$$

for $\sigma>1+\epsilon$ as $y \rightarrow \infty$. Choose $\epsilon=(\sigma-1) / 2$ for simplicity, and we have demonstrated that

$$
Q_{\mathfrak{a} m}(z, s, 1 ; \bar{f}) \ll y_{\Gamma}(z)^{1 / 2-\sigma / 2}
$$

for $\sigma>1$ and an implied constant depending on $\sigma, f$ and $\Gamma$ alone. This is (iii).

Taking derivatives we see, for any $\gamma$ in $P S L_{2}(\mathbb{R})$,

$$
\begin{aligned}
2 i y \frac{d}{d z}\left(\overline{F_{\mathfrak{a}}\left(\sigma_{\mathfrak{a}} \gamma z\right)} \operatorname{Im}(\gamma z)^{s} e(m \gamma z)\right) & =s \overline{F_{\mathfrak{a}}\left(\sigma_{\mathfrak{a}} \gamma z\right)} \operatorname{Im}(\gamma z)^{s} e(m \gamma z) \varepsilon(\gamma, z)^{-2} \\
& -4 \pi m \overline{F_{\mathfrak{a}}\left(\sigma_{\mathfrak{a}} \gamma z\right)} \operatorname{Im}(\gamma z)^{s+1} e(m \gamma z) \varepsilon(\gamma, z)^{-2} .
\end{aligned}
$$

Hence

$$
\begin{aligned}
y Q_{\mathfrak{a} m}^{\prime}(z, s, 1 ; \bar{f}) \ll & |s| \sum_{\gamma \in \Gamma_{\mathfrak{a}} \backslash \Gamma}\left|F_{\mathfrak{a}}(\gamma z)\right| \operatorname{Im}\left(\sigma_{\mathfrak{a}}{ }^{-1} \gamma z\right)^{\sigma} \\
& +|m| \sum_{\gamma \in \Gamma_{\mathfrak{a}} \backslash \Gamma}\left|F_{\mathfrak{a}}(\gamma z)\right| \operatorname{Im}\left(\sigma_{\mathfrak{a}}{ }^{-1} \gamma z\right)^{\sigma+1} \\
\ll & \ll(|m|+1) y_{\Gamma}(z)^{1-\sigma+\epsilon}
\end{aligned}
$$

and set $\epsilon=(\sigma-1) / 2$ as before to obtain (iv).

Finally, it is easy to see that

$$
y G_{\mathfrak{a} m}(z, s ; \bar{F})=\sum_{\gamma \in \Gamma_{\mathfrak{a}} \backslash \Gamma} \overline{F_{\mathfrak{a}}(\gamma z)} \operatorname{Im}\left({\sigma_{\mathfrak{a}}}^{-1} \gamma z\right)^{s+1} e\left(m{\sigma_{\mathfrak{a}}}^{-1} \gamma z\right) \varepsilon\left({\sigma_{\mathfrak{a}}}^{-1} \gamma, z\right)^{-2} .
$$


So the argument used for $Q_{\mathfrak{a} m}(z, s, 1 ; \bar{f})$ applies to $G_{\mathfrak{a} m}(z, s-1 ; \bar{F})$ yielding (v), and the proof is complete.

\section{Proof of Propositions B and C}

Proposition B. For $k \in 2 \mathbb{Z}$ the (Eisenstein) series $U_{\mathfrak{a} 0}(z, s, k)$ has a meromorphic continuation to all $s$ with $\operatorname{Re}(s)>1-\delta_{\Gamma}$. We have

$$
U_{\mathfrak{a} 0}(z, s, k) \ll y_{\Gamma}(z)^{\sigma}
$$

for these $s$ with the implied constant depending on $s, k$ and $\Gamma$. The only possible pole in this region appears at $s=1$ when $k=0$. It is a simple pole with residue $1 / V$.

Proof. The Eisenstein series $E_{\mathfrak{a}}(z, s)=U_{\mathfrak{a} 0}(z, s, 0)$ has a meromorphic continuation to all $s$ in $\mathbb{C}$. This is shown in Chapter 6 of [1]. There it is also shown that the Fourier expansion

$$
E_{\mathfrak{a}}\left(\sigma_{\mathfrak{b}} z, s\right)=\delta_{\mathfrak{a} \mathfrak{b}} y^{s}+\phi_{\mathfrak{a} \mathfrak{b}}(s) y^{1-s}+\sum_{m \neq 0} \phi_{\mathfrak{a} \mathfrak{b}}(m, s) W_{s}(m z)
$$

is valid for all $s$ in $\mathbb{C}$ except at the poles of $E_{\mathfrak{a}}(z, s)$. In particular, for $\operatorname{Re}(s)>1-\delta_{\Gamma}$ the expansion (10.1) is valid except at $s=1$ where $\phi_{\mathfrak{a} \mathfrak{b}}(s)$ has a simple pole. Proposition 6.13 of [1] shows that the residue of $E_{\mathfrak{a}}(z, s)$ at $s=1$ is $1 / V$. The coefficients $\phi_{\mathfrak{a} \mathfrak{b}}(m, s)$ are analytic for $\sigma=\operatorname{Re}(s)>1-\delta_{\Gamma}$ and, for all $s$, satisfy

$$
\phi_{\mathfrak{a} \mathfrak{b}}(m, s) \ll|m|^{\sigma}+|m|^{1-\sigma}
$$

with an implied constant depending uniformly on $s$ (away from the poles) and $\Gamma$. This result is stated in [11, (6.19)] and proved in [JO, Proposition 7.2].

Now by (8.13), (8.14),

$$
\begin{aligned}
R^{n} E_{\mathfrak{a}}(z, s) & =s(s+1) \cdots(s+n-1) U_{\mathfrak{a} 0}(z, s, 2 n), \\
L^{n} E_{\mathfrak{a}}(z, s) & =s(s+1) \cdots(s+n-1) U_{\mathfrak{a} 0}(z, s,-2 n),
\end{aligned}
$$

so we may obtain the meromorphic continuation of $U_{\mathfrak{a} 0}(z, s, k)$ from $E_{\mathfrak{a}}(z, s)$. For example, with Lemma 8.1, Theorem 8.4, (8.18), (10.1) and (10.2) we have

$$
\begin{aligned}
R^{n} E_{\mathfrak{a}}\left(\sigma_{\mathfrak{b}} z, s\right)= & \delta_{\mathfrak{a} \mathfrak{b}} R^{n} y^{s}+\phi_{\mathfrak{a} \mathfrak{b}}(s) R^{n} y^{1-s}+R^{n} \sum_{m \neq 0} \phi_{\mathfrak{a} \mathfrak{b}}(m, s) W_{s}(m z) \\
= & \delta_{\mathfrak{a} \mathfrak{b}} s(s+1) \cdots(s+n-1) y^{s}+\phi_{\mathfrak{a} \mathfrak{b}}(s)(1-s)(2-s) \cdots(n-s) y^{1-s} \\
& \quad+\sum_{m \neq 0} \phi_{\mathfrak{a} \mathfrak{b}}(m, s) R^{n} W_{s}(m z) .
\end{aligned}
$$

For $n>0$ the pole of $\phi_{\mathfrak{a} \mathfrak{b}}(s)$ at 1 is eliminated and we see that

$$
R^{n} E_{\mathfrak{a}}\left(\sigma_{\mathfrak{b}} z, s\right) \ll \delta_{\mathfrak{a} \mathfrak{b}} y^{\sigma}+y^{1-\sigma} .
$$

A similar result holds for $L^{n}$ and we have shown that

$$
U_{\mathfrak{a} 0}\left(\sigma_{\mathfrak{b}} z, s, k\right) \ll \delta_{\mathfrak{a} \mathfrak{b}} y^{\sigma}+y^{1-\sigma}
$$

as $y \rightarrow \infty$ for an implied constant depending on $s, k$ and $\Gamma$. 
Proposition C. For $k \in 2 \mathbb{Z}$ and $m>0$ the Poincaré series $U_{\mathfrak{a} m}(z, s, k)$ has a continuation to an analytic function for all $s$ with $\operatorname{Re}(s)>1-\delta_{\Gamma}$. We have

$$
U_{\mathfrak{a} m}(z, s, k) \ll y_{\Gamma}(z)^{1 / 2}
$$

for these $s$ with the implied constant depending on $s, m, k$ and $\Gamma$.

Proof. We first look at the case $k=0$. In the spectral decomposition (8.1) of $U_{\mathfrak{a} m}(z, s, 0)$ the inner products may be found explicitly as in (8.4) of [JO] and [I3, Chapter 17]:

$$
U_{\mathfrak{a} m}(z, s, 0) \pi^{-1 / 2}(4 \pi m)^{s-1 / 2} \Gamma(s)=\sum_{j=1}^{\infty} \Gamma\left(s-s_{j}\right) \Gamma\left(s-1+s_{j}\right) \overline{\rho_{\mathfrak{a} j}}(m) \eta_{j}(z)
$$

$$
+\frac{1}{4 \pi} \sum_{\mathfrak{b}} \int_{-\infty}^{\infty} \Gamma(s-1 / 2-i r) \Gamma(s-1 / 2+i r) \overline{\phi_{\mathfrak{a} \mathfrak{b}}}(m, 1 / 2+i r) E_{\mathfrak{b}}(z, 1 / 2+i r) d r,
$$

where, referring to $(8.3)$, the $\rho_{\mathfrak{a} j}(m)$ are the Fourier coefficients of the Maass forms $\eta_{j}$. Let

$$
U_{\mathfrak{a} m}(z, s, 0)_{D I S C}=\sum_{j=1}^{\infty} \Gamma\left(s-s_{j}\right) \Gamma\left(s-1+s_{j}\right) \overline{\rho_{\mathfrak{a} j}}(m) \eta_{j}(z),
$$

the discrete spectral component. We shall examine this first. Use (8.5) and (8.7) to get

$$
\Gamma\left(s-s_{j}\right) \Gamma\left(s-1+s_{j}\right) \overline{\rho_{\mathfrak{a} j}}(m) \ll \frac{\left|t_{j}\right|^{2 \sigma-1 / 2}}{\sqrt{|m|}} e^{-\pi\left|t_{j}\right| / 2} .
$$

Lemma 10.1. Let $m$ and $n$ be integers with $m>0, n \geqslant 0$. As functions of $s$, $R^{n}\left(U_{\mathfrak{a} m}(z, s, 0)_{D I S C}\right)$ and $L^{n}\left(U_{\mathfrak{a} m}(z, s, 0)_{D I S C}\right)$ are analytic for $\operatorname{Re}(s)>1-\delta_{\Gamma}$. For these $s$ and an implied constant depending on $s, n$ and $\Gamma$ alone we have

$$
R^{n}\left(U_{\mathfrak{a} m}(z, s, 0)_{D I S C}\right), L^{n}\left(U_{\mathfrak{a} m}(z, s, 0)_{D I S C}\right) \ll|m|^{-1 / 2} y_{\Gamma}(z)^{1 / 2} .
$$

Proof. Let

$$
J(z, s)=\sum_{j=1}^{\infty} \Gamma\left(s-s_{j}\right) \Gamma\left(s-1+s_{j}\right) \overline{\rho_{\mathfrak{a} j}}(m) R^{n}\left(\eta_{j}(z)\right) .
$$

With (10.5), Lemma 8.2 and (8.2) it follows that, for fixed $s$, the series $J(z, s)$ converges uniformly for $z$ in any compact set, say, and is bounded by $|m|^{-1 / 2} y_{\Gamma}(z)^{1 / 2}$. Hence, with Theorem 8.4,

$$
R^{n}\left(U_{\mathfrak{a} m}(z, s, 0)_{D I S C}\right)=J(z, s) .
$$

We also see that $J(z, s)$ converges uniformly for $s$ in compact sets with $\operatorname{Re}(s)>$ $1-\delta_{\Gamma}$ giving an analytic function of $s$ and similarly for $L^{n}$.

To deal with the continuous spectral component,

$$
\begin{aligned}
& U_{\mathfrak{a} m}(z, s, 0)_{C O N T} \\
& \quad=\frac{1}{4 \pi} \sum_{\mathfrak{b}} \int_{-\infty}^{\infty} \Gamma(s-1 / 2-i r) \Gamma(s-1 / 2+i r) \overline{\phi_{\mathfrak{a} \mathfrak{b}}}(m, 1 / 2+i r) E_{\mathfrak{b}}(z, 1 / 2+i r) d r,
\end{aligned}
$$

we shall need the next lemma. 
Lemma 10.2. For $\psi(r)$ smooth on $[T, T+1]$ we have

$$
\frac{d}{d z} \int_{T}^{T+1} \psi(r) E_{\mathfrak{a}}\left(\sigma_{\mathfrak{b}} z, 1 / 2+i r\right) d r=\int_{T}^{T+1} \psi(r)\left(\frac{d}{d z} E_{\mathfrak{a}}\left(\sigma_{\mathfrak{b}} z, 1 / 2+i r\right)\right) d r .
$$

Proof. With the Fourier expansion (4.6) we have

$$
\begin{aligned}
E_{\mathfrak{a}}\left(\sigma_{\mathfrak{b}} z, 1 / 2+i r\right)= & \delta_{\mathfrak{a} \mathfrak{b}} y^{1 / 2+i r}+\phi_{\mathfrak{a} \mathfrak{b}}(1 / 2+i r) y^{1 / 2-i r} \\
& +\sum_{m \neq 0} \phi_{\mathfrak{a} \mathfrak{b}}(m, 1 / 2+i r) W_{1 / 2+i r}(m z) .
\end{aligned}
$$

Combine (8.6), (8.9) and (8.11) to see that

$$
\begin{aligned}
\int_{T}^{T+1} \psi(r) & \left(\delta_{\mathfrak{a} \mathfrak{b}}\left|y^{1 / 2+i r}\right|+\left|\phi_{\mathfrak{a} \mathfrak{b}}(1 / 2+i r) y^{1 / 2-i r}\right|\right. \\
& \left.+\sum_{m \neq 0}\left|\phi_{\mathfrak{a} \mathfrak{b}}(m, 1 / 2+i r) W_{1 / 2+i r}(m z)\right|\right) d r<\infty
\end{aligned}
$$

and hence, by Corollary 8.6,

$$
\begin{aligned}
& \int_{T}^{T+1} \psi(r) E_{\mathfrak{a}}\left(\sigma_{\mathfrak{b}} z, 1 / 2+i r\right) d r=\int_{T}^{T+1} \psi(r)\left(\delta_{\mathfrak{a} \mathfrak{b}} y^{1 / 2+i r}+\phi_{\mathfrak{a} \mathfrak{b}}(1 / 2+i r) y^{1 / 2-i r}\right) d r \\
& (10.6) \quad+\sum_{m \neq 0} \int_{T}^{T+1} \psi(r) \phi_{\mathfrak{a} \mathfrak{b}}(m, 1 / 2+i r) W_{1 / 2+i r}(m z) d r
\end{aligned}
$$

and similarly, using (8.15),

$$
\begin{aligned}
\int_{T}^{T+1} & \psi(r)\left(\frac{d}{d z} E_{\mathfrak{a}}\left(\sigma_{\mathfrak{b}} z, 1 / 2+i r\right)\right) d r \\
= & \int_{T}^{T+1} \psi(r)\left(\delta_{\mathfrak{a} \mathfrak{b}} \frac{d\left(y^{1 / 2+i r}\right)}{d z}+\phi_{\mathfrak{a} \mathfrak{b}}(1 / 2+i r) \frac{d\left(y^{1 / 2-i r}\right)}{d z}\right) d r \\
& +\sum_{m \neq 0} \int_{T}^{T+1} \psi(r) \phi_{\mathfrak{a} \mathfrak{b}}(1 / 2+i r, m)\left(\frac{d}{d z} W_{1 / 2+i r}(m z)\right) d r .
\end{aligned}
$$

To demonstrate that the derivative of (10.6) equals (10.7) we need to show that all the corresponding components are equal. For example,

$$
\frac{d}{d z} \int_{T}^{T+1} \psi(r) y^{\sigma+i r} d r=\int_{T}^{T+1} \psi(r) \frac{d\left(y^{\sigma+i r}\right)}{d z} d r
$$

because, using Theorem 8.5, it is easy to check that, for each $y$,

$$
\left((y+h)^{\sigma+i r}-y^{\sigma+i r}\right) / h
$$

is uniformly bounded for $r$ in $[T, T+1]$ as $h \rightarrow 0$. In the same way,

$$
\frac{d}{d z} \int_{T}^{T+1} \psi(r) W_{1 / 2+i r}(m z) d r=\int_{T}^{T+1} \psi(r)\left(\frac{d}{d z} W_{1 / 2+i r}(m z)\right) d r
$$

completing the proof. 
Moreover, the same arguments and Lemma 8.1 imply

$$
\begin{aligned}
R^{n} \int_{T}^{T+1} \psi(r) E_{\mathfrak{a}}\left(\sigma_{\mathfrak{b}} z, 1 / 2+i r\right) d r & =\int_{T}^{T+1} \psi(r)\left(R^{n} E_{\mathfrak{a}}\left(\sigma_{\mathfrak{b}} z, 1 / 2+i r\right)\right) d r, \\
L^{n} \int_{T}^{T+1} \psi(r) E_{\mathfrak{a}}\left(\sigma_{\mathfrak{b}} z, 1 / 2+i r\right) d r & =\int_{T}^{T+1} \psi(r)\left(L^{n} E_{\mathfrak{a}}\left(\sigma_{\mathfrak{b}} z, 1 / 2+i r\right)\right) d r .
\end{aligned}
$$

Returning to our continuous spectral component,

$$
\begin{aligned}
& R^{n} U_{\mathfrak{a} m}(z, s, 0)_{C O N T} \\
& =\frac{1}{4 \pi} \sum_{\mathfrak{b}} R^{n} \int_{-\infty}^{\infty} \Gamma(s-1 / 2-i r) \Gamma(s-1 / 2+i r) \overline{\phi_{\mathfrak{a} \mathfrak{b}}}(m, 1 / 2+i r) E_{\mathfrak{b}}(z, 1 / 2+i r) d r .
\end{aligned}
$$

If we restrict our attention to $r$ in $[T, T+1]$ we find

$$
\begin{aligned}
& \frac{1}{4 \pi} \sum_{\mathfrak{b}} R^{n} \int_{T}^{T+1} \Gamma(s-1 / 2-i r) \Gamma(s-1 / 2+i r) \overline{\phi_{\mathfrak{a} \mathfrak{b}}}(m, 1 / 2+i r) E_{\mathfrak{b}}(z, 1 / 2+i r) d r \\
& =\frac{1}{4 \pi} \sum_{\mathfrak{b}} \int_{T}^{T+1} \Gamma(s-1 / 2-i r) \Gamma(s-1 / 2+i r) \overline{\phi_{\mathfrak{a} \mathfrak{b}}}(m, 1 / 2+i r) R^{n} E_{\mathfrak{b}}(z, 1 / 2+i r) d r \\
& \ll T e^{-\pi T} \sqrt{\int_{T}^{T+1}\left|\phi_{\mathfrak{a} \mathfrak{b}}(m, 1 / 2+i r)\right|^{2}} d r \sqrt{\int_{T}^{T+1}\left|R^{n} E_{\mathfrak{b}}(z, 1 / 2+i r)\right|^{2} d r} \\
& \ll T^{2 n+8} e^{-\pi T / 2}|m|^{-1 / 2} y_{\Gamma}(z)^{1 / 2},
\end{aligned}
$$

where we used (10.9) to get the second line, the Cauchy-Schwarz inequality and (8.7) for line three, and (8.10) and Lemma 8.3 for the last line. Therefore, repeating the argument for $L^{n}$, we have shown the following.

Lemma 10.3. Let $m$ and $n$ be integers with $m>0, n \geqslant 0$. As functions of $s$, $R^{n}\left(U_{\mathfrak{a} m}(z, s, 0)_{C O N T}\right)$ and $L^{n}\left(U_{\mathfrak{a} m}(z, s, 0)_{C O N T}\right)$ are analytic for $\operatorname{Re}(s)>1-\delta_{\Gamma}$. For these $s$ and an implied constant depending on $s, n$ and $\Gamma$ alone we have

$$
R^{n}\left(U_{\mathfrak{a} m}(z, s, 0)_{C O N T}\right), L^{n}\left(U_{\mathfrak{a} m}(z, s, 0)_{C O N T}\right) \ll|m|^{-1 / 2} y_{\Gamma}(z)^{1 / 2} .
$$

We may now finish the proof of Proposition C. With (8.13) we see that

$$
\begin{aligned}
R_{0} U_{\mathfrak{a} m}(z, s, 0) & =s U_{\mathfrak{a} m}(z, s, 2)-4 \pi m U_{\mathfrak{a} m}(z, s+1,2), \\
R_{2} R_{0} U_{\mathfrak{a} m}(z, s, 0) & =s(s+1) U_{\mathfrak{a} m}(z, s, 4)-4 \pi m(2 s+2) U_{\mathfrak{a} m}(z, s+1,4) \\
& +(4 \pi m)^{2} U_{\mathfrak{a} m}(z, s+2,4) .
\end{aligned}
$$

In general, for $k \geqslant 0$,

$$
\begin{aligned}
& U_{\mathfrak{a} m}(z, s, 2 k)=\frac{1}{s(s+1) \cdots(s+k-1)}\left(R^{k} U_{\mathfrak{a} m}(z, s, 0)\right. \\
& \left.+p_{1}(m, s) U_{\mathfrak{a} m}(z, s+1,2 k)+\cdots+p_{k}(m, s) U_{\mathfrak{a} m}(z, s+k, 2 k)\right)
\end{aligned}
$$

with polynomials $p_{i}$ in $m$ and $s$. Therefore, using Lemmas 10.1, 10.3 and Proposition A, part (ii), the right side of (10.11) is analytic for $\operatorname{Re}(s)>1-\delta_{\Gamma}$ and bounded by $y_{\Gamma}(z)^{1 / 2}$. A similar result holds for $k<0$. 


\section{Proof of Proposition D}

Proposition D. For $m \geqslant 0$, both series $(s-1) Q_{\mathfrak{a} m}(z, s, 1 ; \bar{f})$ and $Q_{\mathfrak{a} m}^{\prime}(z, s, 1 ; \bar{f})$ have continuations to analytic functions of $s$ with $\operatorname{Re}(s)>1-\delta_{\Gamma}$. For these $s$ values,

$$
(s-1) Q_{\mathfrak{a} m}(z, s, 1 ; \bar{f}), \quad y Q_{\mathfrak{a} m}^{\prime}(z, s, 1 ; \bar{f}) \ll y_{\Gamma}(z)^{1 / 2} .
$$

The implied constant depends on $s, m, f$ and $\Gamma$. Also $Q_{\mathfrak{a} m}(z, s, 1 ; \bar{f})$ has a simple pole at $s=1$ with residue $2 i \overline{\left\langle f, P_{\mathfrak{a} m}(\cdot)_{2}\right\rangle}$.

Proof. By Proposition A, part (iii), $Q_{\mathfrak{a} m}(z, s, 1 ; \bar{f})$ is certainly square integrable for $\operatorname{Re}(s)>1$. In other words,

$$
\left\langle Q_{\mathfrak{a} m}(\cdot, s, 1 ; \bar{f}), Q_{\mathfrak{a} m}(\cdot, s, 1 ; \bar{f})\right\rangle<\infty .
$$

The Roelcke-Selberg decomposition, (8.1), yields

$$
\begin{gathered}
Q_{\mathfrak{a} m}(z, s, 1 ; \bar{f})=\sum_{j=0}^{\infty}\left\langle Q_{\mathfrak{a} m}(\cdot, s, 1 ; \bar{f}), \eta_{j}\right\rangle \eta_{j}(z) \\
+\frac{1}{4 \pi} \sum_{\mathfrak{b}} \int_{-\infty}^{\infty}\left\langle Q_{\mathfrak{a} m}(\cdot, s, 1 ; \bar{f}), E_{\mathfrak{b}}(\cdot, 1 / 2+i r)\right\rangle E_{\mathfrak{b}}(z, 1 / 2+i r) d r .
\end{gathered}
$$

To understand the inner products appearing in (11.1), we make use of the next lemma.

Lemma 11.1. Let $\xi_{1}, \xi_{2}$ and $\psi$ be any smooth $\Gamma$ invariant functions (not necessarily in $\left.L^{2}(\Gamma \backslash \mathfrak{H})\right)$. If $(\Delta-\lambda) \xi_{1}=\xi_{2},\left(\Delta-\lambda^{\prime}\right) \psi=0$ and

$$
\begin{aligned}
\xi_{1}, R_{0} \xi_{1}, \Delta \xi_{1} & \ll y_{\Gamma}(z)^{A}, \\
\psi, R_{0} \psi & \ll y_{\Gamma}(z)^{B}
\end{aligned}
$$

for $A+B<0$ and $R_{0}=2 i y \frac{d}{d z}$ the raising operator, then

$$
\left\langle\xi_{1}, \psi\right\rangle=\frac{1}{\lambda^{\prime}-\lambda}\left\langle\xi_{2}, \psi\right\rangle .
$$

Proof. We simply have

$$
\left\langle\xi_{1}, \psi\right\rangle=\frac{1}{\lambda^{\prime}-\lambda}\left\langle\xi_{1},(\Delta-\bar{\lambda}) \psi\right\rangle=\frac{1}{\lambda^{\prime}-\lambda}\left\langle(\Delta-\lambda) \xi_{1}, \psi\right\rangle=\frac{1}{\lambda^{\prime}-\lambda}\left\langle\xi_{2}, \psi\right\rangle .
$$

To justify switching $\Delta$ from the right side of the inner product to the left side requires the growth assumptions we stated. See [JO, Proposition 9.3 and Corollary 9.4] for the proof of this.

Now for all $n \in \mathbb{Z}$,

$$
\begin{aligned}
(\Delta-s(1-s)) Q_{\mathfrak{a} m}(z, s, n ; \bar{f})= & -8 \pi i m Q_{\mathfrak{a} m}(z, s+2, n-1 ; \bar{f}) \\
& +4 \pi m s Q_{\mathfrak{a} m}(z, s+1, n ; \bar{f}) \\
& +2 i s Q_{\mathfrak{a} m}(z, s+1, n-1 ; \bar{f})
\end{aligned}
$$

We want to apply this lemma to $\xi_{1}=Q_{\mathfrak{a} m}(z, s, n ; \bar{f})$ and $\xi_{1}=\eta_{j}$ (recall that $\left.\left(\Delta-s_{j}\left(1-s_{j}\right)\right) \eta_{j}=0\right)$. To check the growth conditions we will need the following result. 
Proposition E. For $-n \leqslant 0$ the series $Q_{\mathfrak{a} m}(z, s+n+1,-n ; \bar{f})$ is an analytic function of $s$ for $\operatorname{Re}(s)>1-\delta_{\Gamma}$. Also for these $s$ we have

$$
Q_{\mathfrak{a} m}(z, s+n+1,-n ; \bar{f}), \quad R_{0} Q_{\mathfrak{a} m}(z, s+n+1,-n ; \bar{f}) \ll e^{-\pi y_{\Gamma}(z)}
$$

with the implied constant depending on $n, m, f, s$ and $\Gamma$ alone.

The proof of this proposition follows at the end of this section and depends on the nice fact that $Q_{\mathfrak{a} m}(z, s+n+1,-n ; \bar{f})$ with $-n \leqslant 0$ can be expressed as a linear combination of Poincaré series $U_{\mathfrak{a} m}(z, s, k)$ multiplied by something with exponential decay at the cusps (that is, $\bar{f}$ with its weight lowered by $L$ ).

Now we have $\eta_{j}(z), R_{0} \eta_{j}(z) \ll y_{\Gamma}(z)^{1 / 2}$ by Lemma 8.2 and

$$
Q_{\mathfrak{a} m}(z, s, 1 ; \bar{f}), R_{0} Q_{\mathfrak{a} m}(z, s, 1 ; \bar{f}), \Delta Q_{\mathfrak{a} m}(z, s, 1 ; \bar{f}) \ll y_{\Gamma}(z)^{1 / 2-\sigma / 2}
$$

for $\sigma=\operatorname{Re}(s)>1$ by Proposition A, parts (iii) and (iv) and Proposition E. So we may use Lemma 11.1 to get, for $\operatorname{Re}(s)>2$,

$$
\begin{aligned}
\left\langle Q_{\mathfrak{a} m}(\cdot, s, 1 ; \bar{f}), \eta_{j}\right\rangle & =\frac{1}{\left(s_{j}-s\right)\left(1-s_{j}-s\right)}\left(-8 \pi i m\left\langle Q_{\mathfrak{a} m}(\cdot, s+2,0 ; \bar{f}), \eta_{j}\right\rangle\right. \\
& \left.+4 \pi m s\left\langle Q_{\mathfrak{a} m}(\cdot, s+1,1 ; \bar{f}), \eta_{j}\right\rangle+2 i s\left\langle Q_{\mathfrak{a} m}(\cdot, s+1,0 ; \bar{f}), \eta_{j}\right\rangle\right) .
\end{aligned}
$$

We can repeat this procedure $W$ times in all to obtain, again for $\operatorname{Re}(s)>2$,

$$
\left\langle Q_{\mathfrak{a} m}(\cdot, s, 1 ; \bar{f}), \eta_{j}\right\rangle=\sum_{l} \frac{P_{l}(m, s)}{R_{l}\left(s_{j}, s\right)}\left\langle Q_{\mathfrak{a} m}\left(\cdot, s+W+c_{l}, 1-d_{l} ; \bar{f}\right), \eta_{j}\right\rangle,
$$

with integers $c_{l}, d_{l}$ satisfying $0 \leqslant c_{l}, d_{l} \leqslant W, d_{l} \leq W+c_{l}, P_{l}(m, s)$ a polynomial in $m, s$ alone of degree $W$ in $m$ and of degree $W$ in $s$ and $R_{l}\left(s_{j}, s\right)$ a polynomial in $s_{j}, s$ alone of degree $2 W$ in $s_{j}$ and of degree $2 W$ in $s$. In fact,

$$
R_{l}\left(s_{j}, s\right)=\prod_{b}\left(s_{j}-b-s\right)\left(1-s_{j}-b-s\right),
$$

where, for each $l$, the product is over some subset of integers $b$ in $\{0,1, \ldots, 2 W\}$ of cardinality $W$.

The finite sum on the right of (11.2) may be used to give the analytic continuation of the inner product on the left and to bound it. For our purposes we are only interested in getting the analytic continuation to $\operatorname{Re}(s)>1-\delta_{\Gamma}$. Examining each term on the right of (11.2) we see that if $d_{l}=0$, then we have

$$
Q_{\mathfrak{a} m}\left(z, s+W+c_{l}, 1 ; \bar{f}\right) \ll y_{\Gamma}(z)^{1 / 4-W / 2}
$$

by Proposition A, (iii) for $W \geqslant 1$. Hence

$$
\left\langle Q_{\mathfrak{a} m}\left(\cdot, s+W+c_{l}, 1 ; \bar{f}\right), \eta_{j}\right\rangle \ll \sqrt{\left\|y_{\Gamma}(z)^{-1 / 4}\right\| \cdot\left\|\eta_{j}\right\|}=\sqrt{\left\|y_{\Gamma}(z)^{-1 / 4}\right\|} \ll 1 .
$$

For $0<d_{l} \leqslant W$, Proposition E implies that

$$
Q_{\mathfrak{a} m}\left(z, s+W+c_{l}, 1-d_{l} ; \bar{f}\right) \ll e^{-\pi y_{\Gamma}(z)} .
$$

Hence, as in (8.6),

$$
\left\langle Q_{\mathfrak{a} m}\left(z, s+W+c_{l}, 1-d_{l} ; \bar{f}\right), \eta_{j}\right\rangle \ll 1 .
$$


Now combine (11.2), (11.3), (11.5) and (11.7) to see that, for $j>0,\left\langle Q_{\mathfrak{a} m}(\cdot, s, 1 ; \bar{f})\right.$, $\left.\eta_{j}\right\rangle$ is an analytic function of $s$ for $\operatorname{Re}(s)>1-\delta_{\Gamma}$ and satisfies

$$
\left\langle Q_{\mathfrak{a} m}(\cdot, s, 1 ; \bar{f}), \eta_{j}\right\rangle \ll\left|s_{j}\right|^{-2 W} \ll\left|\lambda_{j}\right|^{-W}
$$

for implied constants depending on $s, m, W, f$ and $\Gamma$ alone.

For $j>0$ we can use (8.2), (8.8) and (11.8) to get

$$
\sum_{T \leqslant\left|\lambda_{j}\right|<T+1}\left\langle Q_{\mathfrak{a} m}(\cdot, s, 1 ; \bar{f}), \eta_{j}\right\rangle \eta_{j}(z) \ll T\left[y_{\Gamma}(z)^{1 / 2}+T^{7 / 4} y_{\Gamma}(z)^{-3 / 2}\right] T^{-W} .
$$

Therefore (using any $W \geqslant 4$ ),

$$
\sum_{j=1}^{\infty}\left\langle Q_{\mathfrak{a} m}(\cdot, s, 1 ; \bar{f}), \eta_{j}\right\rangle \eta_{j}(z) \ll y_{\Gamma}(z)^{1 / 2}
$$

for all $s$ with $\operatorname{Re}(s)>1-\delta_{\Gamma}$ and an implied constant depending solely on $s, m, f$ and $\Gamma$.

For $j=0$ the constant eigenfunction is $\eta_{0}=V^{-1 / 2}$. By unfolding we obtain

$$
\begin{aligned}
\left\langle Q_{\mathfrak{a} m}(\cdot, s, 1 ; \bar{f}), \eta_{0}\right\rangle \eta_{0} & =\frac{-\overline{a_{\mathfrak{a}}(m)} \Gamma(s-1)}{2 \pi i m(4 \pi m)^{s-1}} \\
& =\frac{-\overline{a_{\mathfrak{a}}(m)}}{2 \pi i m}\left(\frac{1}{s-1}+O(1)\right) \\
& =2 i \overline{\left\langle f, P_{\mathfrak{a} m}(\cdot)_{2}\right\rangle}\left(\frac{1}{s-1}+O(1)\right)
\end{aligned}
$$

as $s \rightarrow 1$ since $\left\langle f, P_{\mathfrak{a} m}(\cdot)_{2}\right\rangle=a_{\mathfrak{a}}(m) /(4 \pi m)$ for

$$
f_{\mathfrak{a}}(z)=j\left(\sigma_{\mathfrak{a}}, z\right)^{-2} f\left(\sigma_{\mathfrak{a}} z\right)=\sum_{m=1}^{\infty} a_{\mathfrak{a}}(m) e(m z) .
$$

With arguments similar to those used for the discrete spectrum we now consider the continuous spectrum. For $P_{l}, R_{l}, c_{l}$ and $d_{l}$ identical to (11.2),

$$
\begin{aligned}
& \left\langle Q_{\mathfrak{a} m}(\cdot, s, 1 ; \bar{f}), E_{\mathfrak{b}}(\cdot, 1 / 2+i r)\right\rangle \\
& \quad=\sum_{l} \frac{P_{l}(m, s)}{R_{l}(1 / 2+i r, s)}\left\langle Q_{\mathfrak{a} m}\left(\cdot, s+W+c_{l}, 1-d_{l} ; \bar{f}\right), E_{\mathfrak{b}}(\cdot, 1 / 2+i r)\right\rangle,
\end{aligned}
$$

which is true for $\operatorname{Re}(s)>2$ initially. Here we employed (4.6), (8.6), (8.9) and (8.11) to get the bound

$$
E_{\mathfrak{a}}(z, 1 / 2+i r) \ll y_{\Gamma}(z)^{1 / 2}
$$

for $r \in[T, T+1]$ and an implied constant depending on $T$ and $\Gamma$.

With (11.4), (11.6) and (11.12) we see that (for $W \geqslant 1$ ) the right side of (11.11) converges and gives the analytic continuation of the left side to $\operatorname{Re}(s)>1-\delta_{\Gamma}$. 
Now

$$
\begin{gathered}
\int_{T}^{T+1}\left\langle Q_{\mathfrak{a} m}(\cdot, s, 1 ; \bar{f}), E_{\mathfrak{b}}(\cdot, 1 / 2+i r)\right\rangle E_{\mathfrak{b}}\left(z_{0}, 1 / 2+i r\right) d r \\
=\sum_{l} P_{l}(m, s) \int_{T}^{T+1} \frac{\left\langle Q_{\mathfrak{a} m}\left(\cdot, s+W+c_{l}, 1-d_{l} ; \bar{f}\right), E_{\mathfrak{b}}\left(\cdot, \frac{1}{2}+i r\right)\right\rangle}{R_{l}(1 / 2+i r, s)} E_{\mathfrak{b}}\left(z_{0}, \frac{1}{2}+i r\right) d r
\end{gathered}
$$

$$
=\sum_{l} P_{l}(m, s) \int_{T}^{T+1} \int_{\mathfrak{F}} \frac{Q_{\mathfrak{a} m}\left(z, s+W+c_{l}, 1-d_{l} ; \bar{f}\right)}{R_{l}(1 / 2+i r, s)} \overline{E_{\mathfrak{b}}\left(z, \frac{1}{2}+i r\right)} E_{\mathfrak{b}}\left(z_{0}, \frac{1}{2}+i r\right) d \mu z d r .
$$

The integrand satisfies

$$
\begin{aligned}
\frac{Q_{\mathfrak{a} m}\left(z, s+W+c_{l}, 1-d_{l} ; \bar{f}\right)}{R_{l}(1 / 2+i r, s)} & \overline{E_{\mathfrak{b}}(z, 1 / 2+i r)} E_{\mathfrak{b}}\left(z_{0}, 1 / 2+i r\right) \\
& \ll|r|^{-2 W} y_{\Gamma}(z)^{1 / 4-W / 2} y_{\Gamma}(z)^{1 / 2} y_{\Gamma}\left(z_{0}\right)^{1 / 2}
\end{aligned}
$$

by (11.3), (11.4), (11.6) and (11.12). Thus the double integral in (11.13) is absolutely and uniformly convergent and we may interchange the limits of integration to obtain

$\sum_{l} P_{l}(m, s) \int_{\mathfrak{F}} Q_{\mathfrak{a} m}\left(z, s+W+c_{l}, 1-d_{l} ; \bar{f}\right) \int_{T}^{T+1} \frac{\overline{E_{\mathfrak{b}}(z, 1 / 2+i r)}}{R_{l}(1 / 2+i r, s)} E_{\mathfrak{b}}\left(z_{0}, \frac{1}{2}+i r\right) d r d \mu z$.

Also

$$
\begin{aligned}
& \int_{T}^{T+1} \frac{\overline{E_{\mathfrak{b}}(z, 1 / 2+i r)}}{R_{l}(1 / 2+i r, s)} E_{\mathfrak{b}}\left(z_{0}, 1 / 2+i r\right) d r \\
& \ll T^{-2 W} \sqrt{\int_{T}^{T+1}\left|E_{\mathfrak{b}}(z, 1 / 2+i r)\right|^{2} d r \cdot \int_{T}^{T+1}\left|E_{\mathfrak{b}}\left(z_{0}, 1 / 2+i r\right)\right|^{2} d r .}
\end{aligned}
$$

So, with Lemma 8.3 (for $n=0$ ), (11.14) is bounded by a constant times

$$
\begin{array}{r}
\sum_{l}\left|P_{l}(m, s)\right| \int_{\mathfrak{F}}\left|Q_{\mathfrak{a} m}\left(z, s+W+c_{l}, 1-d_{l} ; \bar{f}\right)\right| T^{-2 W} y_{\Gamma}(z)^{1 / 2} T^{6} y_{\Gamma}\left(z_{0}\right)^{1 / 2} T^{6} d \mu z \\
\ll \sum_{l}\left|P_{l}(m, s)\right| T^{12-2 W} \int_{\mathfrak{F}} y_{\Gamma}(z)^{3 / 4-W / 2} d \mu z \cdot y_{\Gamma}\left(z_{0}\right)^{1 / 2} .
\end{array}
$$

This means that, for $W$ chosen large enough,

$$
\int_{-\infty}^{\infty}\left\langle Q_{\mathfrak{a} m}(\cdot, s, 1 ; \bar{f}), E_{\mathfrak{b}}(\cdot, 1 / 2+i r)\right\rangle E_{\mathfrak{b}}(z, 1 / 2+i r) d r \ll y_{\Gamma}(z)^{1 / 2} .
$$

Combine this with (11.9) and (11.10) to see that $Q_{\mathfrak{a} m}(z, s, 1 ; \bar{f})$ is analytic for $\operatorname{Re}(s)>1-\delta_{\Gamma}$ and bounded by $y_{\Gamma}(z)^{1 / 2}$ except for a simple pole at $s=1$ with the stated residue.

We leave it to the reader to check the result for $R_{0} Q_{\mathfrak{a} m}(z, s, 1 ; \bar{f})$ by applying $R_{0}$ to both sides of (11.1) and using the estimates from Section 8 and Proposition E. Note that $R_{0}$ eliminates the pole at $s=1$ coming from $\eta_{0}$, the constant eigenfunction. This completes the proof of Proposition D. 
Proof of Proposition E. We begin with the formula

$$
\overline{f^{(n)}(\gamma z)}=(-2 i)^{-n} \operatorname{Im}(\gamma z)^{-n-1} \sum_{r=0}^{n}(-1)^{n-r} \varepsilon(\gamma, z)^{-2 r-2}\left(\begin{array}{l}
n \\
r
\end{array}\right) \frac{(n+1) !}{(r+1) !} L^{r}(y \overline{f(z)})
$$

for $f$ in $S_{2}(\Gamma)$ and $\gamma$ in $\Gamma$. This formula may be proved by induction; see [C].

Then, by definition, $I_{\mathfrak{a}(-n)}(z)=f_{\mathfrak{a}}^{(n)}(z)$ for $-n \leqslant 0$ and

$$
Q_{\mathfrak{a} m}(z, s,-n ; \bar{f})=\sum_{\gamma \in \Gamma_{\mathfrak{a}} \backslash \Gamma} \overline{f_{\mathfrak{a}}^{(n)}\left(\sigma_{\mathfrak{a}}^{-1} \gamma z\right)} \operatorname{Im}\left(\sigma_{\mathfrak{a}}^{-1} \gamma z\right)^{s} e\left(m \sigma_{\mathfrak{a}}{ }^{-1} \gamma z\right) .
$$

So, if we name $\Gamma^{\prime}={\sigma_{\mathfrak{a}}}^{-1} \Gamma \sigma_{\mathfrak{a}}$ and note that ${\sigma_{\mathfrak{a}}}^{-1} \Gamma_{\mathfrak{a}} \sigma_{\mathfrak{a}}=\Gamma_{\infty}$, we find

$$
\begin{aligned}
& Q_{\mathfrak{a} m}\left(\sigma_{\mathfrak{a}} z, s,-n ; \bar{f}\right)= \sum_{\gamma^{\prime} \in \Gamma_{\infty} \backslash \Gamma^{\prime}} \overline{f_{\mathfrak{a}}^{(n)}\left(\gamma^{\prime} z\right)} \operatorname{Im}\left(\gamma^{\prime} z\right)^{s} e\left(m \gamma^{\prime} z\right) \\
&=(-2 i)^{-n} \sum_{r=0}^{n}(-1)^{n-r}\left(\begin{array}{l}
n \\
r
\end{array}\right) \frac{(n+1) !}{(r+1) !} L^{r}\left(y \overline{f_{\mathfrak{a}}(z)}\right) \\
& \times \sum_{\gamma^{\prime} \in \Gamma_{\infty} \backslash \Gamma^{\prime}} \operatorname{Im}\left(\gamma^{\prime} z\right)^{s-n-1} e\left(m \gamma^{\prime} z\right) \varepsilon\left(\gamma^{\prime}, z\right)^{-2 r-2} \\
&=(-2 i)^{-n} \sum_{r=0}^{n}(-1)^{n-r}\left(\begin{array}{l}
n \\
r
\end{array}\right) \frac{(n+1) !}{(r+1) !} L^{r}\left(y \overline{f_{\mathfrak{a}}(z)}\right) \\
& \times \varepsilon\left(\sigma_{\mathfrak{a}}, z\right)^{-2 r-2} U_{\mathfrak{a} m}\left(\sigma_{\mathfrak{a}} z, s-n-1,2 r+2\right) .
\end{aligned}
$$

Thus, recalling (8.20), (8.21),

$$
\begin{aligned}
L^{r}\left(\overline{f_{\mathfrak{a}}(z)}\right) & =L^{r} \theta_{\sigma_{\mathfrak{a}},-2}(y \overline{f(z)}) \\
& =\theta_{\sigma_{\mathfrak{a}},-2 r-2} L^{r}(y \overline{f(z)}) \\
& =\left.L^{r}(y \overline{f(z)})\right|_{\sigma_{\mathfrak{a}} z} \varepsilon\left(\sigma_{\mathfrak{a}}, z\right)^{2 r+2} .
\end{aligned}
$$

So we get

$$
Q_{\mathfrak{a} m}(z, s,-n ; \bar{f})=(-2 i)^{-n} \sum_{r=0}^{n}(-1)^{n-r}\left(\begin{array}{l}
n \\
r
\end{array}\right) \frac{(n+1) !}{(r+1) !} L^{r}(y \overline{f(z)}) U_{\mathfrak{a} m}(z, s-n-1,2 r+2) .
$$

This identity (11.15) provides the analytic continuation of $Q_{\mathfrak{a} m}(z, s,-n ; \bar{f})$ to $\operatorname{Re}(s)$ $>2+n-\delta_{\Gamma}$ by Propositions B and C.

The piece $L^{r}(y \overline{f(z)})$ has exponential decay at every cusp $\mathfrak{b}$ because

$$
\begin{aligned}
\theta_{\sigma_{\mathfrak{b}},-2 r-2} L^{r}(y \overline{f(z)}) & =L^{r}\left(\theta_{\sigma_{\mathfrak{b}},-2} y \overline{f(z)}\right) \\
& =L^{r}\left(y \overline{j\left(\sigma_{\mathfrak{b}} z\right)^{-2} f\left(\sigma_{\mathfrak{b}} z\right)}\right) \\
& =L^{r}\left(y \sum_{n=1}^{\infty} \overline{a_{\mathfrak{b}}(n) e(n z)}\right) .
\end{aligned}
$$


Hence

$$
L^{r}(y \overline{f(z)}) \ll y_{\Gamma}(z)^{r+1} e^{-2 \pi y_{\Gamma}(z)}
$$

for an implied constant depending on $r, f$ and $\Gamma$. Therefore, with (11.15), (11.16), Propositions B and C, we have

$$
Q_{\mathfrak{a} m}(z, s+n+1,-n ; \bar{f}) \ll e^{-\pi y_{\Gamma}(z)},
$$

say, for $\operatorname{Re}(s)>1-\delta_{\Gamma}$.

To show that the same results are true for $R_{0} Q_{\mathfrak{a} m}(z, s+n+1,-n ; \bar{f})$, apply $R_{0}$ to both sides of (11.15) and note that

$$
\begin{aligned}
& R_{0}\left(L^{r}(y \overline{f(z)}) U_{\mathfrak{a} m}(z, s-n-1,2 r+2)\right) \\
& \begin{array}{r}
=\left(R_{-2 r-2} L^{r}(y \overline{f(z)})\right) U_{\mathfrak{a} m}(z, s-n-1,2 r+2) \\
\quad+L^{r}(y \overline{f(z)}) R_{2 r+2} U_{\mathfrak{a} m}(z, s-n-1,2 r+2) \\
\quad=R_{-2 r-2} L^{r}(y \overline{f(z)}) U_{\mathfrak{a} m}(z, s-n-1,2 r+2) \\
+L^{r}(y \overline{f(z)})\left((s-n+r) U_{\mathfrak{a} m}(z, s-n-1,2 r+4)-4 \pi m U_{\mathfrak{a} m}(z, s-n, 2 r+4)\right)
\end{array}
\end{aligned}
$$

by (8.13). This completes the proof of Proposition E.

\section{Further QUESTIONS}

Many natural questions arise:

(1) We have found the dimensions of the spaces $S_{k}^{2}(\Gamma)$ and $M_{k}^{2}(\Gamma)$ focusing on even weight $k$. What are the dimensions for $k$ odd (as in Theorem 2.25 in [Sh] where the dimensions of $S_{k}(\Gamma)$ and $M_{k}(\Gamma)$ are given) or for $\Gamma \backslash \mathfrak{H}$ compact?

(2) As we saw in (3.12), forms with characters arise naturally in the work of Kleban and Zagier. All the spaces we have discussed may be generalized to arbitrary weights and multiplier systems.

(3) What are the Fourier coefficients of these second-order forms and do they have arithmetic or other significance?

(4) What is the dimension of $R_{k}^{2}(\Gamma)$ ? It is certainly true by Remark 5.5 that

$$
\operatorname{dim} S_{2}^{2}(\Gamma)<\operatorname{dim} R_{2}^{2}(\Gamma) \leqslant \operatorname{dim} M_{2}^{2}(\Gamma) .
$$

(5) Is there a natural inner product on the spaces $S_{k}^{2}(\Gamma)$ and $M_{k}^{2}(\Gamma)$ that respects (3.10) and (3.11)?

(6) A further interesting extension of this work is to higher order forms. We purposely designed our notation in Section 2 wih this in mind. Define the third-order space $S_{k}^{3}(\Gamma)$ with the conditions $\mathbf{H}, \mathbf{A}\left(S_{k}^{2}(\Gamma)\right), \mathbf{P}$ and $\mathbf{C}$. Recursively, $S_{k}^{n}(\Gamma)$ should satisfy $\mathbf{H}, \mathbf{A}\left(S_{k}^{n-1}(\Gamma)\right), \mathbf{P}$ and $\mathbf{C}$ so that the automorphy condition involves a form of lower order. A similar result holds for the higher order versions of the other spaces in Section 2. We expect that the methods used in this paper should generalize to counting dimensions of these higher order spaces. 


\section{ACKNOWLEDGEMENTS}

The authors thank R. Bruggeman and M. Knopp for their very useful comments and suggestions.

\section{REFERENCES}

[B] K. Brown, Cohomology of groups, Graduate Texts in Mathematics, vol. 87, SpringerVerlag (1982). MR672956 (83k:20002)

[CDO] G. Chinta, N. Diamantis, C. O'Sullivan, Second order modular forms, Acta Arith. 103 (2002), 209-223. MR1905087(2003b:11037)

[CO] G. Chinta, C. O'Sullivan, Poincaré series constructed from period polynomials (to appear).

[DI] F. Diamond, J. Im, Modular forms and modular curves., In Seminar on Fermat's Last Theorem, Providence, RI, 1995, pp. 39-133.. MR.1357209 (97g:11044)

[DKMO] N. Diamantis, M. Knopp, G. Mason, C. O'Sullivan, L-functions of second-order cusp forms, Ramanujan J. 12 (2006), 327-347. MR2293794 (2008c:11073)

[DO] N. Diamantis, C. O'Sullivan, Hecke theory of series formed with modular symbols and relations among convolution L-functions, Mathematische Annalen 318 (1) (2000), 85105. MR 1785577 (2002e:11063)

[F] D. Farmer, Converse theorems and second order modular forms, AMS sectional meeting talk, Salt Lake City, 2002.

[FW] D. Farmer, K. Wilson, Converse theorems assuming a partial Euler product, arXiv:math. NT/0408221v1 (2004).

[G] D. Goldfeld, Zeta functions formed with modular symbols, Proc. of the Symposia in Pure Math. 66 (1999), 111-122. MR1703748 (2000g:11039)

[GO] D. Goldfeld, C. O'Sullivan, Estimating additive character sums for Fuchsian groups, Ramanujan J. 7 (2003), 241-267. MR2035805 (2005c:11060)

[Gu] R. C. Gunning, The Eichler cohomology groups and automorphic forms, Trans. Amer. Math. Soc. 100 (1961), 44-62. MR0140126 (25:3549)

[I1] H. Iwaniec, Spectral methods of automorphic forms, 2nd ed., vol. 53, Graduate Studies in Mathematics, Amer. Math. Soc., 2002. MR1942691 (2003k:11085)

[I2] H. Iwaniec, Topics in Classical Automorphic Forms, vol. 17, Graduate Studies in Mathematics, Amer. Math. Soc., 1997. MR1474964 (98e:11051)

[I3] H. Iwaniec, Fourier coefficients of modular forms and Kloosterman sums, Unpublished lecture notes, Rutgers University (1987).

[IM] O. Imamoglu, Y. Martin, A converse theorem for second-order modular forms of level $N$, Acta Arith. 1234 (2006), 361-376. MR2262250 (2008a:11061)

[JO] J. Jorgenson, C. O'Sullivan., Convolution Dirichlet series and a Kronecker limit formula for second-order Eisenstein series, Nagoya Math. J. 179 (2005), 47-102. MR.2164401 (2006k:11080)

[K] M. Knopp, Some new results on the Eichler cohomology of automorphic forms, Bull. Amer. Math. Soc. 80 (1974), 607-632. MR 0344454 (49:9193)

[KZ] P. Kleban, D. Zagier, Crossing probabilities and modular forms, J. Stat. Phys. 113 (2003), 431-454. MR2013692 (2004j:82018)

[O1] C. O'Sullivan, Properties of Eisenstein series formed with modular symbols, J. Reine Angew. Math. 518 (2000), 163-186. MR1739405 (2000j:11073)

[O2] C. O'Sullivan, Identities from the holomorphic projection of modular forms, Number Theory for the Millennium III (2002), 87-106. MR.1956270 (2003m:11064)

[PR] Y. N. Petridis, M. S. Risager, Modular symbols have a normal distribution, Geom. Funct. Anal. 14 (2004), 1013-1043. MR2105951 (2005h:11101)

[Ra] J. Ratcliffe, Foundations of Hyperbolic Manifolds, Springer-Verlag, New York, 1994. MR1299730 (95j:57011)

[Ru] W. Rudin, Principles of Mathematical Analysis, McGraw-Hill, 1964. MR0166310 $(29: 3587)$

[Sa] P. Sarnak, Some Applications of Modular Forms, Cambridge Tracts in Math. 99, Cambridge Univ. Press, 1990. MR,1102679 (92k:11045) 
[Se] A. Selberg, On Discontinuous Groups in Higher-dimensional Spaces, Tata Institute, Bombay (1960). MR0130324 (24:A188)

[Sh] G. Shimura, Introduction to the Arithmetic Theory of Automorphic Functions, Princeton Univ. Press, Princeton, NJ, 1971. MR0314766 (47:3318)

Department of Mathematics, University of Nottingham, Nottingham, England

Department of Mathematics and Computer Science, Bronx Community College, Bronx, New York 10453 